\title{
Weak solutions of the Cahn-Hilliard system with dynamic boundary conditions: A gradient flow approach
}

\author{
Harald Garcke and Patrik Knopf \\ Fakultät für Mathematik, Universität Regensburg, 93053 Regensburg, Germany \\ Harald.Garcke@ur.de, Patrik.Knopf@ur.de \\ This is a preprint version of the paper. Please cite as: \\ H. Garcke and P. Knopf, SIAM J. Math. Anal. 52-1 (2020), pp. 340-369. \\ https://doi.org/10.1137/19M1258840
}

\begin{abstract}
The Cahn-Hilliard equation is the most common model to describe phase separation processes of a mixture of two components. For a better description of short-range interactions of the material with the solid wall, various dynamic boundary conditions have been considered in recent times. New models with dynamic boundary conditions have been proposed recently by $\mathrm{C}$. Liu and $\mathrm{H}$. Wu [6]. We prove the existence of weak solutions to these new models by interpreting the problem as a suitable gradient flow of a total free energy which contains volume as well as surface contributions. The formulation involves an inner product which couples bulk and surface quantities in an appropriate way. We use an implicit time discretization and show that the obtained approximate solutions converge to a weak solution of the Cahn-Hilliard system. This allows us to substantially improve earlier results which needed strong assumptions on the geometry of the domain. Furthermore, we prove that this weak solution is unique.
\end{abstract}

Keywords: Cahn-Hilliard equation, dynamic boundary conditions, gradient flow.

MSC Classification: 35A01, 35A02, 35A15

\section{Introduction}

The Cahn-Hilliard equation was originally derived to model spinodal decomposition in binary alloys. Later it was noticed that the Cahn-Hilliard equations also describes later stages of the evolution of phase transition phenomena like Ostwald ripening. Here a particular important aspect of the Cahn-Hilliard equation is that topological changes in the interface can be handled directly. This is in contrast to a classical free boundary description where singularities occur in cases where the topology changes. Later new application of the Cahn-Hilliard model appeared in the literature in such areas as imaging sciences, non-isothermal phase transitions and two-phase flow. In certain applications it also turned out to be essential to model boundary effects more accurately. In order to do so several dynamic boundary conditions have been proposed in the literature which we are going to review in the following. In this paper we will focus on a new dynamic boundary condition proposed recently by [6] which is in particular important for hydrodynamic applications. It is expected that these boundary conditions will have an important impact on the correct modeling of contact line phenomena. Let us now describe the models studied in the literature in more detail.

Let $T$ and $\varepsilon$ be positive real numbers and let $\Omega \subset \mathbb{R}^{d}$ (where $d \in\{2,3\}$ ) be a bounded domain with boundary $\Gamma:=\partial \Omega$. The unit outer normal vector on $\Gamma$ will be denoted by $\mathbf{n}=\mathbf{n}(x)$. Then the standard Cahn-Hilliard equation (cf. [7]) reads as follows:

$$
\begin{aligned}
\partial_{t} \phi & =\Delta \mu, \\
\mu & =-\varepsilon \Delta \phi+\frac{1}{\varepsilon} F^{\prime}(\phi) .
\end{aligned}
$$

Usually, this equation is endowed with an initial condition

$$
\left.\phi\right|_{t=0}=\phi_{0} .
$$


Here, $\phi=\phi(x, t)$ and $\mu=\mu(x, t)$ are functions that depend on time $t \geq 0$ and position $x \in \Omega$. The symbol $\partial_{t}$ denotes the partial derivative with respect to time and $\Delta$ is the Laplace operator that acts on the variables $x \in \Omega$. The phase field $\phi$ represents the difference $\phi_{1}-\phi_{2}$ of two local relative concentrations $\phi_{i}, i=1,2$. This means that the regions $\{x \in \Omega: \phi(x)=1\}$ and $\{x \in \Omega: \phi(x)=-1\}$ correspond to the pure phases of the materials while $\{x \in \Omega:-1<\phi(x)<1\}$ represents the diffuse interface between them. The thickness of this interface is proportional to the parameter $\varepsilon>0$. Therefore, $\varepsilon$ is usually chosen very small. The chemical potential $\mu$ can be understood as the Fréchet derivative of the bulk free energy

$$
E_{\text {bulk }}(\phi)=\int_{\Omega} \frac{\varepsilon}{2}|\nabla \phi|^{2}+\frac{1}{\varepsilon} F(\phi) \mathrm{d} x .
$$

The function $F$ represents the bulk potential which typically is of double well form. A common choice is the smooth double well potential

$$
W_{\mathrm{dw}}(\phi)=\theta\left(\phi^{2}-1\right)^{2}, \quad \theta>0
$$

which has two minima at $\phi= \pm 1$ and a local (unstable) maximum at $\phi=0$. The time-evolution of $\phi$ is considered in a bounded domain and hence suitable boundary conditions have to be imposed. The homogeneous Neumann conditions

$$
\begin{array}{ll}
\partial_{\mathbf{n}} \mu=0 & \text { on } \Gamma \times] 0, T[ \\
\partial_{\mathbf{n}} \phi=0 & \text { on } \Gamma \times] 0, T[
\end{array}
$$

are the classical choice. From the no-flux condition (1.4) we can deduce mass conservation in the bulk

$$
\int_{\Omega} \phi(t) \mathrm{d} x=\int_{\Omega} \phi(0) \mathrm{d} x, \quad t \in[0, T]
$$

and conditions (1.4),(1.5) imply that the bulk free energy is decreasing in the following way:

$$
\left.\frac{\mathrm{d}}{\mathrm{d} t} E_{\text {bulk }}(\phi(t))+\int_{\Omega}|\nabla \mu(t)|^{2} \mathrm{~d} x=0, \quad t \in\right] 0, T[.
$$

The Cahn-Hilliard equation (1.1a),(1.1b) with the initial condition (1.1c) and the homogeneous Neumann conditions (1.4) and (1.5) is already very well understood. It has been investigated from many different viewpoints, for instance see [1, 5, 9, 16, 17, 22, 23, 29, 31, 36].

However, especially for certain materials, the ansatz (1.5) is not satisfactory as it neglects certain additional influences of the boundary to the dynamics in the bulk. For a better description of interactions between the wall and the mixture, physicists introduced a surface free energy functional given by

$$
E_{\text {surf }}(\phi)=\int_{\Gamma} \frac{\kappa \varepsilon}{2}\left|\nabla_{\Gamma} \phi\right|^{2}+\frac{1}{\varepsilon} G(\phi) \mathrm{d} S
$$

where $\nabla_{\Gamma}$ denotes the surface gradient operator on $\Gamma, G$ is a surface potential and $\kappa$ is a nonnegative parameter that is related to interfacial effects at the boundary. In the case $\kappa=0$ the problem is related to the moving contact line problem that is studied in [33]. Moreover, several dynamic boundary conditions have been proposed in the literature to replace the homogeneous Neumann conditions (see, e.g., [10, 12, 15, 25, 26, 27, 28, 30, 35]). Results for the Allen-Cahn equation (which is another phase-field equation, cf. [2]) with dynamic boundary conditions can be found, for instance, in [11, 13, 14].

We give some concrete examples of dynamic boundary conditions for the Cahn-Hilliard equation:

- The boundary condition

$$
\left.\varepsilon \partial_{t} \phi=\kappa \varepsilon \Delta_{\Gamma} \phi-\varepsilon \partial_{\mathbf{n}} \phi-\frac{1}{\varepsilon} G^{\prime}(\phi) \quad \text { on } \Gamma \times\right] 0, T[
$$

was suggested (e.g. in [24]) to replace the condition (1.5) and analyzed for example in [8]. The symbol $\Delta_{\Gamma}$ denotes the Laplace-Beltrami operator on $\Gamma$. Equation (1.9) is a surface Allen-Cahn equation which has $\partial_{\mathbf{n}} \phi$ as an additional source term. 
- The coupled boundary condition

$$
\left\{\begin{aligned}
\partial_{t} \phi & =\sigma \Delta_{\Gamma} \mu-\partial_{\mathbf{n}} \mu & & \text { on } \Gamma \times] 0, T[, \\
\mu & =-\kappa \varepsilon \Delta_{\Gamma} \phi+\varepsilon \partial_{\mathbf{n}} \phi+\frac{1}{\varepsilon} G^{\prime}(\phi) & & \text { on } \Gamma \times] 0, T[
\end{aligned}\right.
$$

for some $\sigma \geq 0$ was proposed in [18, 22] to replace both (1.4) and (1.5). In the case $\sigma=0$ (which was first proposed in [18]), this type of dynamic boundary condition is called a Wentzell boundary condition. Furthermore, the first line of (1.10) directly implies that the total (i.e., bulk plus boundary) mass is conserved.

- Another very generic boundary condition to replace (1.5) is

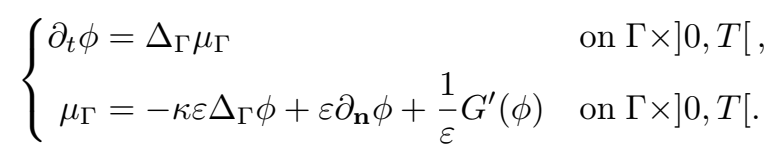

It was derived in [6] by an energetic variational approach which is based on the following physical principles: Separate conservation of mass both in the bulk and on the surface, dissipation of the total free energy (that is $E:=E_{\text {bulk }}+E_{\text {surf }}$ ) and the force balance both in the bulk and on the boundary.

Similar to the situation in the bulk, the chemical potential on the boundary $\mu_{\Gamma}$ can be described by the Fréchet derivative of the surface free energy $E_{\text {surf }}$. However, the interaction term $\varepsilon \partial_{\mathbf{n}} \phi$ must be added. We point out that $\mu_{\Gamma}$ does not necessarily coincide with the trace of $\mu$ on $\Gamma$ but is to be understood as an independent variable.

A mathematical correspondence between the above dynamic boundary conditions and the total free energy

$$
E(\phi)=E_{\mathrm{bulk}}(\phi)+E_{\mathrm{surf}}(\phi)
$$

will be explained in Section 3.

In this paper, we investigate the Cahn-Hilliard system subject to the dynamic boundary condition (1.11). This means that the overall system reads as follows:

$$
\begin{aligned}
& \left.\partial_{t} \phi=\Delta \mu \quad \text { with } \quad \mu=-\varepsilon \Delta \phi+\frac{1}{\varepsilon} F^{\prime}(\phi) \quad \text { in } \Omega \times\right] 0, T[, \\
& \begin{array}{ll}
\partial_{\mathbf{n}} \mu=0 & \text { on } \Gamma \times] 0, T[,
\end{array} \\
& \left.\partial_{t} \phi=\Delta_{\Gamma} \mu_{\Gamma} \quad \text { with } \quad \mu_{\Gamma}=-\kappa \varepsilon \Delta_{\Gamma} \phi+\varepsilon \partial_{\mathbf{n}} \phi+\frac{1}{\varepsilon} G^{\prime}(\phi) \quad \text { on } \Gamma \times\right] 0, T[\text {, } \\
& \phi(\cdot, 0)=\phi_{0} \quad \text { in } \Omega .
\end{aligned}
$$

If a solution of (1.13) is sufficiently regular, it directly follows that the mass in the bulk and the mass on the surface are conserved:

$$
\int_{\Omega} \phi(t) \mathrm{d} x=\int_{\Omega} \phi(0) \mathrm{d} x \quad \text { and } \quad \int_{\Gamma} \phi(t) \mathrm{d} S=\int_{\Gamma} \phi(0) \mathrm{d} S, \quad t \in[0, T] .
$$

Moreover, the total free energy is still decreasing in time in the following sense:

$$
\left.\frac{\mathrm{d}}{\mathrm{d} t} E(\phi(t))+\int_{\Omega}|\nabla \mu(t)|^{2} \mathrm{~d} x+\int_{\Gamma}\left|\nabla_{\Gamma} \mu_{\Gamma}(t)\right|^{2} \mathrm{~d} x=0, \quad t \in\right] 0, T[.
$$

Existence and uniqueness of weak and strong solutions of the system (1.13) have been established by C. $\mathrm{Liu}$ and $\mathrm{H}$. Wu in [6]. The idea of their proof is to construct solutions of a regularized system where the equations for $\mu$ and $\mu_{\Gamma}$ are replaced by

$$
\mu=-\varepsilon \Delta \phi+\alpha \partial_{t} \phi+\frac{1}{\varepsilon} F^{\prime}(\phi) \quad \text { and } \quad \mu_{\Gamma}=-\kappa \varepsilon \Delta_{\Gamma} \phi+\alpha \partial_{t} \phi+\varepsilon \partial_{\mathbf{n}} \phi+\frac{1}{\varepsilon} G^{\prime}(\phi) .
$$


Then a solution of the original problem can be found by taking the limit $\alpha \rightarrow 0$. However, in the case $\kappa=0$, their proof requires very strong assumptions on the domain $\Omega$ and its boundary $\Gamma$. To be precise, they need the requirement

$$
c_{\mathcal{R}}|\Gamma|^{\frac{1}{2}}|\Omega|^{-1}<1
$$

where $c_{\mathcal{R}}$ is a constant that results from the inverse trace theorem.

In this paper, we use a different ansatz to construct weak solutions of the system (1.13). In our approach the condition (1.16) is not necessary which is a substantial improvement. However, on the other hand, our notion of a weak solution (cf. Definition 4.1) is slightly weaker than that in [6] so both results have their merits. We will show in Section 3 that (1.13) can be regarded as a gradient flow equation of the total free energy, namely

$$
\left\langle\partial_{t} \phi, \eta\right\rangle=-\frac{\delta E}{\delta \phi}(\phi)[\eta]
$$

for all admissible test functions $\eta$ where $\langle\cdot, \cdot\rangle$ is a suitable inner product on a certain function space. This representation can be used to construct a weak solution of the system (1.13) by implicit time discretization which will be done in Section 4. In Section 5, we will establish a uniqueness result for the obtained weak solution. Finally, in Section 6, we present several plots of two numerical simulations. In particular, they demonstrate the influence of mass conservation on the boundary. For simplicity and since it does not play any role in the analysis, we will set $\varepsilon=1$ in Sections 3-5.

\section{Preliminaries}

In this section we introduce some preliminaries that we will use in the rest of this paper:

(P1) In the existence and uniqueness results (see Sections 4 and 5 ), $\Omega \subset \mathbb{R}^{d}$ (with $d \in\{2,3\}$ ) denotes a bounded domain with Lipschitz boundary $\Gamma:=\partial \Omega$. In Sections 1-3, however, we assume additionally that $\Omega$ has a $C^{2}$-boundary. $\mathbf{n}$ denotes the unit outer normal vector on $\Gamma$ and $\partial_{\mathbf{n}}$ is the normal derivative on $\Gamma$. We denote by $|\Omega|$ the $d$-dimensional Lebesgue measure of $\Omega$ and by $|\Gamma|$ the $(d-1)$-dimensional surface measure of $\Gamma$. For any $s>0$ we will also write $\left.\Omega_{s}:=\Omega \times\right] 0, s\left[\right.$ and $\left.\Gamma_{s}:=\Gamma \times\right] 0, s[$.

(P2) We assume that the potentials $F$ and $G$ are bounded from below by

$$
F(\varphi) \geq C_{F} \quad \text { and } \quad G(\varphi) \geq C_{G}, \quad \text { for all } \varphi \in \mathbb{R}
$$

with constants $C_{F}, C_{G} \in \mathbb{R}$. Moreover, we assume that $F$ and $G$ can be written as

$$
F(\varphi)=F_{1}(\varphi)+F_{2}(\varphi) \quad \text { and } \quad G(\varphi)=G_{1}(\varphi)+G_{2}(\varphi), \quad \text { for all } \varphi \in \mathbb{R}
$$

where

(P2.1) $F_{1}, F_{2}, G_{1}, G_{2} \in C^{1}(\mathbb{R})$,

(P2.2) $F_{1}$ and $G_{1}$ are convex and nonnegative,

(P2.3) For any $\delta>0$ there exists constants $A_{F}^{\delta}, A_{G}^{\delta} \geq 0$ such that

$$
\left|F_{1}^{\prime}(\varphi)\right| \leq \delta F_{1}(\varphi)+A_{F}^{\delta} \quad \text { and } \quad\left|G_{1}^{\prime}(\varphi)\right| \leq \delta G_{1}(\varphi)+A_{G}^{\delta}
$$

for all $\varphi \in \mathbb{R}$.

(P2.4) There exist constants $B_{F}, B_{G} \geq 0$ such that

$$
\left|F_{2}^{\prime}(\varphi)\right| \leq B_{F}(|\varphi|+1) \quad \text { and } \quad\left|G_{2}^{\prime}(\varphi)\right| \leq B_{G}(|\varphi|+1)
$$

for all $\varphi \in \mathbb{R}$. 
(P3) For any Banach space $X$, its norm will be denoted by $\|\cdot\|_{X}$. The symbol $\langle\cdot, \cdot\rangle_{X^{*}, X}$ denotes the dual pairing of $X$ and its dual space $X^{*}$. If $X$ is a Hilbert space, its inner product is denoted by $(\cdot, \cdot)_{X}$.

(P4) For any $1 \leq p \leq \infty, L^{p}(\Omega)$ and $L^{p}(\Gamma)$ stand for the Lebesgue spaces that are equipped with the standard norms $\|\cdot\|_{L^{p}(\Omega)}$ and $\|\cdot\|_{L^{p}(\Gamma)}$. For $s \geq 0$ and $1 \leq p \leq \infty$, the symbols $W^{s, p}(\Omega)$ and $W^{s, p}(\Gamma)$ denote the Sobolev spaces with corresponding norms $\|\cdot\|_{W^{s, p}(\Omega)}$ and $\|\cdot\|_{W^{s, p}(\Gamma)}$. Note that $W^{0, p}$ can be identified with $L^{p}$. All Lebesgue spaces and Sobolev spaces are Banach spaces and if $p=2$, they are Hilbert spaces. In this case we will write $H^{s}(\Omega)=W^{s, 2}(\Omega)$ and $H^{s}(\Gamma)=W^{s, 2}(\Gamma)$.

(P5) In general, we will use the symbol $\left.\right|_{\Gamma}$ to denote the trace operator. If $1 \leq p \leq \infty, s>\frac{1}{p}$ and $s-\frac{1}{p}$ is not an integer, the trace operator is uniquely determined and lies in $\mathcal{L}\left(W^{s, p}(\Omega) ; W^{s-1 / p, p}(\Gamma)\right)$, i.e., it is a linear and bounded operator from $W^{s, p}(\Omega)$ to $W^{s-1 / p, p}(\Gamma)$. For brevity, we will sometimes write $\varphi$ instead of $\left.\varphi\right|_{\Gamma}$ if it is clear that we are referring to the trace of $\varphi$.

(P6) By $H^{1}(\Omega)^{*}$ and $H^{1}(\Gamma)^{*}$ we denote the dual spaces of $H^{1}(\Omega)$ and $H^{1}(\Gamma)$. For functions $\varphi \in H^{1}(\Omega)^{*}$ and $\psi \in H^{1}(\Omega)^{*}$ we denote their generalized average by

$$
\langle\varphi\rangle_{\Omega}:=\frac{1}{|\Omega|}\langle\varphi, 1\rangle_{H^{1}(\Omega)^{*}, H^{1}(\Omega)} \quad \text { and } \quad\langle\psi\rangle_{\Gamma}:=\frac{1}{|\Gamma|}\langle\psi, 1\rangle_{H^{1}(\Gamma)^{*}, H^{1}(\Gamma)} .
$$

If $\varphi \in L^{2}(\Omega)$ and $\psi \in L^{2}(\Gamma)$ the above formulas reduce to

$$
\langle\varphi\rangle_{\Omega}=\frac{1}{|\Omega|} \int_{\Omega} \varphi(x) \mathrm{d} x \quad \text { and } \quad\langle\psi\rangle_{\Gamma}=\frac{1}{|\Gamma|} \int_{\Gamma} \psi(x) \mathrm{d} S(x) .
$$

(P7) For any function $\varphi \in H^{1}(\Omega)^{*}$ with $\langle\varphi\rangle_{\Omega}=0$, the Neumann problem

$$
-\Delta \mu=\varphi \quad \text { in } \Omega, \quad \partial_{\mathbf{n}} \mu=0 \quad \text { on } \Gamma
$$

has a unique weak solution $\mu \in H^{1}(\Omega)$ with $\langle\mu\rangle_{\Omega}=0$. For any $\psi \in H^{1}(\Gamma)^{*}$ with $\langle\psi\rangle_{\Gamma}=0$ the equation

$$
-\Delta_{\Gamma} \nu=\psi \quad \text { on } \Gamma
$$

has a unique weak solution $\nu \in H^{1}(\Gamma)$ with $\langle\nu\rangle_{\Gamma}=0$. Here, the symbol $\Delta_{\Gamma}$ denotes the LaplaceBeltrami operator. We will write

$$
(-\Delta)^{-1} \varphi:=\mu \quad \text { and } \quad\left(-\Delta_{\Gamma}\right)^{-1} \psi:=\nu
$$

to denote the above weak solutions.

(P8) In this paper, the spaces $H^{1}(\Omega)^{*}$ and $H^{1}(\Gamma)^{*}$ will be endowed with the following norms (that are equivalent to the standard norm on these spaces):

$$
\begin{aligned}
& \|\varphi\|_{H^{1}(\Omega)^{*}}^{2}=\left\|\nabla(-\Delta)^{-1}\left(\varphi-\langle\varphi\rangle_{\Omega}\right)\right\|_{L^{2}(\Omega)}^{2}+\left|\langle\varphi\rangle_{\Omega}\right|^{2}, \\
& \|\psi\|_{H^{1}(\Gamma)^{*}}^{2}=\left\|\nabla_{\Gamma}\left(-\Delta_{\Gamma}\right)^{-1}\left(\psi-\langle\psi\rangle_{\Gamma}\right)\right\|_{L^{2}(\Gamma)}^{2}+\left|\langle\psi\rangle_{\Gamma}\right|^{2} .
\end{aligned}
$$

(P9) We define the following sets:

$$
\begin{aligned}
& \mathcal{V}^{\kappa}:= \begin{cases}\left\{\varphi \in H^{1}(\Omega)|\varphi|_{\Gamma} \in H^{1}(\Gamma)\right\}, & \kappa>0, \\
H^{1}(\Omega), & \kappa=0,\end{cases} \\
& \mathcal{V}_{m}^{\kappa}:=\left\{\varphi \in \mathcal{V}^{\kappa} \mid\langle\varphi\rangle_{\Omega}=m_{1} \text { and }\langle\varphi\rangle_{\Gamma}=m_{2}\right\}, \quad m=\left(m_{1}, m_{2}\right) \in \mathbb{R}^{2}, \\
& \mathcal{V}_{0}^{\kappa}:=\mathcal{V}_{(0,0)}^{\kappa} .
\end{aligned}
$$


Then $\mathcal{V}^{\kappa}$ is a Hilbert-space with respect to the inner product

$$
(\varphi, \psi)_{\mathcal{V}^{\kappa}}:= \begin{cases}(\varphi, \psi)_{H^{1}(\Omega)}+(\varphi, \psi)_{H^{1}(\Gamma)}, & \kappa>0 \\ (\varphi, \psi)_{H^{1}(\Omega)}, & \kappa=0\end{cases}
$$

and its induced norm $\|\varphi\|_{\mathcal{V}^{\kappa}}:=(\varphi, \varphi)_{\mathcal{V}^{\kappa}}^{1 / 2}$.

For $\varphi \in \mathcal{V}_{0}^{\kappa *}, \mathcal{V}_{0}^{\kappa *}$ being the dual space of $\mathcal{V}_{0}^{\kappa}$, there exists a mean value free $u_{\Omega \varphi} \in H^{1}(\Omega)$ and mean value free $u_{\Gamma, \varphi} \in H^{1}(\Gamma)$ such that for all $\zeta \in \mathcal{V}_{0}^{\kappa}$

$$
\int_{\Omega} \nabla u_{\Omega, \varphi} \cdot \nabla \zeta \mathrm{d} x+\int_{\Gamma} \nabla_{\Gamma} u_{\Gamma, \varphi} \cdot \nabla_{\Gamma} \zeta \mathrm{d} S=\varphi(\zeta) .
$$

Choosing functions $u_{\Omega, \psi}$ and $u_{\Gamma, \psi}$ for $\psi \in \mathcal{V}_{0}^{\kappa *}$ an analogous way, we can define an inner product on $\mathcal{V}_{0}^{\kappa *}$ by

$$
\langle\varphi, \psi\rangle_{\mathcal{V}_{0}^{\kappa *}}=\int_{\Omega} \nabla u_{\Omega, \varphi} \cdot \nabla u_{\Omega, \psi} \mathrm{d} x+\int_{\Gamma} \nabla_{\Gamma} u_{\Gamma, \varphi} \cdot \nabla_{\Gamma} u_{\Gamma, \psi} \mathrm{d} S
$$

We also define its induced norm $\|\varphi\|_{\mathcal{V}_{0}^{\kappa *}}:=\langle\varphi, \varphi\rangle_{\mathcal{V}_{0}^{\kappa *}}^{1 / 2}$. Since $\mathcal{V}_{0}^{\kappa} \subset \mathcal{V}_{0}^{\kappa *}$ we can use this inner product and its induced norm also for functions in $\mathcal{V}_{0}^{\kappa}$. For any $\varphi \in \mathcal{V}_{0}^{\kappa}$, the functions $u_{\Omega, \varphi}$ and $u_{\Gamma, \varphi}$ can be expressed by

$$
u_{\Omega, \varphi}=(-\Delta)^{-1} \varphi \text { and } \quad u_{\Gamma, \varphi}=\left.\left(-\Delta_{\Gamma}\right)^{-1} \varphi\right|_{\Gamma} .
$$

It even holds that $\|\cdot\|_{\mathcal{V}_{0}^{\kappa *}}$ is a norm on $\mathcal{V}_{0}^{\kappa}$ but, of course, $\mathcal{V}_{0}^{\kappa}$ is not complete with respect to this norm.

Remark 2.1. (i) In dealing with weak solutions of the system (1.13) (cf. Sections 4 and 5) it suffices to demand that the domain $\Omega$ has merely a Lipschitz-boundary. However, this is not enough to describe (1.13) pointwisely as the Laplace-Beltrami operator is involved. Therefore, a $C^{2}$-boundary is demanded in the general assumption (P1) .

(ii) One can easily see that, according to (P2), the double well potential

$$
W_{d w}(\phi)=\theta\left(\phi^{2}-1\right)^{2}, \quad \theta>0
$$

is a suitable choice for F or G. However, the logarithmic potential

$$
\begin{array}{r}
W_{\text {log }}(\phi)=\frac{\theta}{2}((1-\phi) \ln (1-\phi)+(1-\phi) \ln (1-\phi))+\frac{\theta_{c}}{2}\left(1-\phi^{2}\right), \\
0<\theta_{c}<\theta,
\end{array}
$$

(which is defined only for $\phi \in]-1,1[$ ) or the obstacle potential

$$
W_{\text {obst }}(\phi)=\left\{\begin{array}{ll}
\theta\left(1-\phi^{2}\right), & \phi \in[-1,1], \\
\infty, & \text { else, }
\end{array} \quad \theta>0,\right.
$$

cannot be chosen as they do not satisfy the condition (P2).

(iii) Any nonnegative, convex, continuously differentiable function $\varphi \mapsto F_{1}(\varphi)$ (or $\varphi \mapsto G_{1}(\varphi)$ respectively) which grows polynomially to $+\infty$ as $|\varphi| \rightarrow \infty$ fulfills (P2.3). However, exponential growth is not allowed, see [21]. 


\section{The gradient flow structure}

For simplicity, we set $\varepsilon=1$. Provided that $\phi, \mu$ and $\mu_{\Gamma}$ are sufficiently regular, we can use the inner product $\langle\cdot, \cdot\rangle_{\mathcal{V}_{0}^{\kappa *}}$ that was introduced in (P9) to describe system (1.13) as a gradient flow equation of the total free energy:

$$
\left\langle\partial_{t} \phi, \eta\right\rangle_{\mathcal{V}_{0}^{\kappa *}}=-\frac{\delta E}{\delta \phi}(\phi)[\eta] \quad \text { for all } \eta \in \mathcal{V}_{0}^{\kappa} \cap L^{\infty}(\Omega),\left.\eta\right|_{\Gamma} \in L^{\infty}(\Gamma)
$$

This holds because for any $\eta \in \mathcal{V}_{0}^{\kappa} \cap L^{\infty}(\Omega)$ with $\left.\eta\right|_{\Gamma} \in L^{\infty}(\Gamma)$,

$$
\begin{aligned}
\left\langle\partial_{t} \phi, \eta\right\rangle_{\mathcal{V}_{0}^{\kappa *}} & =\int_{\Omega} \nabla(-\Delta)^{-1} \partial_{t} \phi \cdot \nabla(-\Delta)^{-1} \eta \mathrm{d} x+\int_{\Gamma} \nabla_{\Gamma}\left(-\Delta_{\Gamma}\right)^{-1} \partial_{t} \phi \cdot \nabla_{\Gamma}\left(-\Delta_{\Gamma}\right)^{-1} \eta \mathrm{d} S \\
& =-\int_{\Omega} \nabla \mu \cdot \nabla(-\Delta)^{-1} \eta \mathrm{d} x-\int_{\Gamma} \nabla_{\Gamma} \mu_{\Gamma} \cdot \nabla_{\Gamma}\left(-\Delta_{\Gamma}\right)^{-1} \eta \mathrm{d} S
\end{aligned}
$$

since $\partial_{t} \phi=\Delta \mu$ in $\Omega_{T}$ and $\partial_{t} \phi=\Delta_{\Gamma} \mu$ on $\Gamma_{T}$. Integration by parts (recall that $\partial_{\mathbf{n}} \mu=0$ ) and the definition of the chemical potentials $\mu$ and $\mu_{\Gamma}$ imply that

$$
\begin{aligned}
\left\langle\partial_{t} \phi, \eta\right\rangle_{\mathcal{V}_{0}^{\kappa *}} & =-\int_{\Omega} \mu \eta \mathrm{d} x-\int_{\Gamma} \mu_{\Gamma} \eta \mathrm{d} S \\
& =-\int_{\Omega}\left(-\Delta \phi+F^{\prime}(\phi)\right) \eta \mathrm{d} x-\int_{\Gamma}\left(-\kappa \Delta_{\Gamma} \phi+\partial_{\mathbf{n}} \phi+G^{\prime}(\phi)\right) \eta \mathrm{d} S \\
& =-\frac{\delta E}{\delta \phi}(\phi)[\eta] .
\end{aligned}
$$

This formal computation shows that the gradient flow equation (3.1) corresponds to the Cahn-Hilliard equation with dynamic boundary conditions given by (1.13). Formally speaking, we can say that this gradient flow is of type $H^{-1}$ both in the bulk and on the surface. However, replacing the inner product $\langle\cdot, \cdot\rangle_{\mathcal{V}_{0}^{\kappa *}}$ in the gradient flow equation (3.1) for the energy $E$ by a different inner product leads to a different $\mathrm{PDE}$ in $\Omega$ and different boundary conditions on $\Gamma$. We give some examples which also can be identified with a gradient flow equation by a similar computation:

(i) The Allen-Cahn equation

$$
\partial_{t} \phi=\Delta \phi-F^{\prime}(\phi) \quad \text { in } \Omega_{T}
$$

with the dynamic boundary condition

$$
\partial_{t} \phi=\kappa \Delta_{\Gamma} \phi-\partial_{\mathbf{n}} \phi-G^{\prime}(\phi) \quad \text { on } \Gamma_{T}
$$

is the gradient flow equation of the energy $E$ with respect to the inner product

$$
\langle\phi, \psi\rangle:=\int_{\Omega} \phi \psi \mathrm{d} x+\int_{\Gamma} \phi \psi \mathrm{d} S .
$$

Formally speaking, the gradient flow is of type $L^{2}$ both in the bulk and on the surface.

(ii) The Allen-Cahn equation

$$
\partial_{t} \phi=\Delta \phi-F^{\prime}(\phi) \quad \text { in } \Omega_{T}
$$

with the dynamic boundary condition

$$
\left\{\begin{aligned}
\partial_{t} \phi & =\Delta_{\Gamma} \mu_{\Gamma} & & \text { on } \Gamma_{T} \\
\mu_{\Gamma} & =-\kappa \Delta_{\Gamma} \phi+\partial_{\mathbf{n}} \phi+G^{\prime}(\phi) & & \text { on } \Gamma_{T}
\end{aligned}\right.
$$


is the gradient flow equation of the energy $E$ with respect to the inner product

$$
\langle\phi, \psi\rangle:=\int_{\Omega} \phi \psi \mathrm{d} x+\int_{\Gamma} \nabla_{\Gamma}\left(-\Delta_{\Gamma}\right)^{-1} \phi \cdot \nabla_{\Gamma}\left(-\Delta_{\Gamma}\right)^{-1} \psi \mathrm{d} S .
$$

This type of system has been analyzed, e.g., in [11]. Formally speaking, the gradient flow is of type $L^{2}$ in the bulk and of type $H^{-1}$ on the surface. By this boundary condition, the mass on the surface is conserved.

(iii) The Cahn-Hilliard equation

$$
\left\{\begin{aligned}
\partial_{t} \phi & =\Delta \mu & & \text { in } \Omega_{T} \\
\mu & =-\Delta \phi+F^{\prime}(\phi) & & \text { in } \Omega_{T}
\end{aligned}\right.
$$

with the homogeneous Neumann condition $\partial_{\mathbf{n}} \mu=0$ on $\Gamma_{T}$ and the dynamic boundary condition

$$
\partial_{t} \phi=\kappa \Delta_{\Gamma} \phi-\partial_{\mathbf{n}} \phi-G^{\prime}(\phi) \quad \text { on } \Gamma_{T}
$$

is the gradient flow equation of the energy $E$ with respect to the inner product

$$
\langle\phi, \psi\rangle:=\int_{\Omega} \nabla(-\Delta)^{-1} \phi \cdot \nabla(-\Delta)^{-1} \psi \mathrm{d} x+\int_{\Gamma} \phi \psi \mathrm{d} S .
$$

This problem is introduced in [24] and analyzed in [15]. Formally speaking, the gradient flow is of type $H^{-1}$ in the bulk and of type $L^{2}$ on the surface. Note that the boundary condition $\partial_{\mathbf{n}} \mu=0$ leads to mass conservation in the bulk.

(iv) Let us now consider the elliptic system

$$
\left\{\begin{aligned}
\Delta u & =f_{1} & & \text { in } \Omega, \\
\sigma \Delta_{\Gamma} u-\partial_{\mathbf{n}} u & =f_{2} & & \text { on } \Gamma .
\end{aligned}\right.
$$

Using the Lax-Milgram theorem one can show that the system (3.2) with $f=\left(f_{1}, f_{2}\right)$ has a unique weak solution $u=\mathcal{S}(f)$ with $\langle u\rangle_{\Omega}=0$ if the right-hand side $f$ satisfies the conditions $\left\langle f_{1}\right\rangle_{\Omega}+\left\langle f_{2}\right\rangle_{\Gamma}=0$. This means that we can define a solution operator $f \mapsto \mathcal{S}(f)$ that maps any admissible right-hand side $f$ onto its corresponding solution.

Then, the Cahn-Hilliard equation

$$
\left\{\begin{aligned}
\partial_{t} \phi & =\Delta \mu & & \text { in } \Omega_{T} \\
\mu & =-\Delta \phi+F^{\prime}(\phi) & & \text { in } \Omega_{T}
\end{aligned}\right.
$$

with the dynamic boundary condition

$$
\left\{\begin{aligned}
\partial_{t} \phi & =\sigma \Delta_{\Gamma} \mu-\partial_{\mathbf{n}} \mu & & \text { on } \Gamma_{T} \\
\mu & =-\kappa \Delta_{\Gamma} \phi+\partial_{\mathbf{n}} \phi+G^{\prime}(\phi) & & \text { on } \Gamma_{T}
\end{aligned}\right.
$$

(for some parameter $\sigma \geq 0$ ) is the gradient flow equation of the energy $E$ with respect to the inner product

$$
\langle\phi, \psi\rangle:=\int_{\Omega} \nabla \mathcal{S}(\phi) \cdot \nabla \mathcal{S}(\psi) \mathrm{d} x+\sigma \int_{\Gamma} \nabla_{\Gamma} \mathcal{S}(\phi) \cdot \nabla_{\Gamma} \mathcal{S}(\psi) \mathrm{d} S .
$$

This is the problem introduced in $[18,22]$ which reduces to the Wentzell boundary condition for $\sigma=0$ (see $[11,18,19])$. In this model, the total mass (i.e., the sum of bulk and surface mass) is conserved. Note that integrating and adding the second lines of (3.3) and (3.4) implies that

$$
\int_{\Omega} \mu \mathrm{d} x+\int_{\Gamma} \mu \mathrm{d} S=\int_{\Omega} F^{\prime}(\phi) \mathrm{d} x+\int_{\Gamma} G^{\prime}(\phi) \mathrm{d} S
$$


must hold. Therefore, $\mu$ cannot be equal to $S\left(\partial_{t} \phi\right)$ but instead $\mu=S\left(\partial_{t} \phi\right)+c$ where the constant is given by

$$
c:=\frac{\int_{\Omega} F^{\prime}(\phi) \mathrm{d} x+\int_{\Gamma} G^{\prime}(\phi) \mathrm{d} S}{|\Omega|+|\Gamma|} .
$$

Then $\mu$ is still a solution of (3.2) with $f_{1}=\partial_{t} \phi$ and $f_{2}=\left.\partial_{t} \phi\right|_{\Gamma}$ and the inner product is not affected by this shift as only the gradients of the solution components are involved.

In the next section we will exploit the gradient flow structure (3.1) to construct a weak solution of the Cahn-Hilliard system (1.13) by implicit time discretization. Certainly, weak solutions of the above systems (i)-(iv) could be constructed in a similar fashion.

\section{Existence of a weak solution}

As stated in the introduction, we consider the following Cahn-Hilliard system with a dynamic boundary condition with $\varepsilon=1$ :

$$
\begin{array}{llll}
\partial_{t} \phi=\Delta \mu & \text { with } & \mu=-\Delta \phi+F^{\prime}(\phi) & \text { in } \Omega \times] 0, T[, \\
\partial_{\mathbf{n}} \mu=0 & & & \text { on } \Gamma \times] 0, T[, \\
\partial_{t} \phi=\Delta_{\Gamma} \mu_{\Gamma} & \text { with } & \mu_{\Gamma}=-\kappa \Delta_{\Gamma} \phi+\partial_{\mathbf{n}} \phi+G^{\prime}(\phi) & \text { on } \Gamma \times] 0, T[, \\
\phi(\cdot, 0)=\phi_{0} & & & \text { in } \Omega .
\end{array}
$$

The choice $\varepsilon=1$ means no loss of generality as it does not play any role in the analysis.

\subsection{Weak solutions and the existence theorem}

Before formulating the existence theorem we give the definition of a weak solution of the Cahn-Hilliard equation (4.1). In the following, as we only consider weak solutions, it suffices to assume that $\Omega$ has merely a Lipschitz-boundary.

Definition 4.1. Let $T \in] 0, \infty\left[, \kappa \geq 0\right.$ and let $\phi_{0} \in \mathcal{V}_{m}^{\kappa}$ be any initial datum having finite energy, i.e., $E\left(\phi_{0}\right)<\infty$. Then the triple $\left(\phi, \mu, \mu_{\Gamma}\right)$ is called a weak solution of the system (4.1) if the following holds:

(i) The occuring functions have the following regularity:

$$
\begin{aligned}
& \phi \in C\left([0, T] ; L^{2}(\Omega)\right) \cap L^{\infty}\left(0, T ; H^{1}(\Omega)\right), \quad \mu \in L^{2}\left(0, T ; H^{1}(\Omega)\right), \\
& \left.\phi\right|_{\Gamma} \in C\left([0, T] ; L^{2}(\Gamma)\right) \cap\left\{\begin{array}{ll}
L^{\infty}\left(0, T ; H^{1}(\Gamma)\right), & \kappa>0, \\
L^{\infty}\left(0, T ; H^{1 / 2}(\Gamma)\right), & \kappa=0,
\end{array} \mu_{\Gamma} \in L^{2}\left(0, T ; H^{1}(\Gamma)\right),\right. \\
& F^{\prime}(\phi) \in L^{1}\left(\Omega_{T}\right), \quad G^{\prime}(\phi) \in L^{1}\left(\Gamma_{T}\right) .
\end{aligned}
$$

(ii) The following weak formulations are satisfied:

$$
\int_{\Omega_{T}}\left(\phi-\phi_{0}\right) \partial_{t} \zeta \mathrm{d}(x, t)=\int_{\Omega_{T}} \nabla \mu \cdot \nabla \zeta \mathrm{d}(x, t)
$$

for all $\zeta \in L^{2}\left(0, T ; H^{1}(\Omega)\right)$ with $\partial_{t} \zeta \in L^{2}\left(\Omega_{T}\right)$ and $\zeta(T)=0$,

$$
\int_{\Gamma_{T}}\left(\phi-\phi_{0}\right) \partial_{t} \xi \mathrm{d}(x, t)=\int_{\Gamma_{T}} \nabla_{\Gamma} \mu_{\Gamma} \cdot \nabla_{\Gamma} \xi \mathrm{d}(x, t)
$$


for all $\xi \in L^{2}\left(0, T ; H^{1}(\Gamma)\right)$ with $\partial_{t} \xi \in L^{2}\left(\Gamma_{T}\right)$ and $\xi(T)=0$ and

$$
\begin{aligned}
\int_{\Omega_{T}} \mu \eta \mathrm{d} x+\int_{\Gamma_{T}} \mu_{\Gamma} \eta \mathrm{d} S \mathrm{~d} t= & \int_{\Omega_{T}} \nabla \phi \cdot \nabla \eta \mathrm{d}(x, t)+\int_{\Omega_{T}} F^{\prime}(\phi) \eta \mathrm{d}(x, t) \\
& +\int_{\Gamma_{T}} \kappa \nabla_{\Gamma} \phi \cdot \nabla_{\Gamma} \eta \mathrm{d} S \mathrm{~d} t+\int_{\Gamma_{T}} G^{\prime}(\phi) \eta \mathrm{d} S \mathrm{~d} t
\end{aligned}
$$

for all $\eta \in L^{2}\left(0, T ; \mathcal{V}^{\kappa}\right) \cap L^{\infty}\left(\Omega_{T}\right)$ with $\left.\eta\right|_{\Gamma} \in L^{\infty}(\Gamma)$.

(iii) The energy inequality is satisfied, i.e., for all $t \in[0, T]$,

$$
E(\phi(t))+\frac{1}{2} \int_{0}^{t}\|\nabla \mu(s)\|_{L^{2}(\Omega)}^{2}+\left\|\nabla_{\Gamma} \mu_{\Gamma}(s)\right\|_{L^{2}(\Gamma)}^{2} \mathrm{~d} s \leq E\left(\phi_{0}\right)<\infty .
$$

Remark 4.2. Let us assume that $\left(\phi, \mu, \mu_{\Gamma}\right)$ is a weak solution in the sense of Definition 4.1.

(a) It follows from the weak formulations (4.2) and (4.3) that

$$
\begin{aligned}
\langle\phi(t)\rangle_{\Omega} & =\left\langle\phi_{0}\right\rangle_{\Omega}=m_{1}, \\
\langle\phi(t)\rangle_{\Gamma} & =\left\langle\phi_{0}\right\rangle_{\Gamma}=m_{2}
\end{aligned}
$$

for all $t \in[0, T]$. To prove this, let $\left.\left.t_{0} \in\right] 0, T\right]$ and $0<h<t_{0} / 2$ be arbitrary and choose

$$
\zeta(t):= \begin{cases}1, & t<t_{0}-h \\ \left(t_{0}-t\right) / h, & t_{0}-h \leq t \leq t_{0} \\ 0, & t>t_{0}\end{cases}
$$

Then $\zeta$ is a suitable test function with $\nabla \zeta=0$ and $\partial_{t} \zeta=-\frac{1}{h} \chi_{\left[t_{0}-h, t_{0}\right]}$. Plugging $\zeta$ into (4.2) yields

$$
\frac{1}{h} \int_{t_{0}-h}^{t_{0}} \int_{\Omega} \phi(t) \mathrm{d} x \mathrm{~d} t=\int_{\Omega} \phi_{0} \mathrm{~d} x
$$

Since $t \mapsto \int_{\Omega} \phi(t) \mathrm{d} x$ is continuous, (4.5) follows as $h$ tends to zero. The assertion (4.6) can be proved analogously.

(b) Since $\mu \in L^{2}\left(0, T ; H^{1}(\Omega)\right)$ and $\mu_{\Gamma} \in L^{2}\left(0, T ; H^{1}(\Gamma)\right)$, it also follows (by definition) from the weak formulations (4.2) and (4.3) that $\phi$ and $\left.\phi\right|_{\Gamma}$ have generalized derivatives with respect to $t$ given by

$$
\partial_{t} \phi=\Delta \mu \text { in } L^{2}\left(0, T ; H^{1}(\Omega)^{*}\right),\left.\quad \partial_{t} \phi\right|_{\Gamma}=\Delta_{\Gamma} \mu_{\Gamma} \text { in } L^{2}\left(0, T ; H^{1}(\Gamma)^{*}\right) .
$$

The following theorem constitutes the main result of this paper: The existence of a weak solution of the Cahn-Hilliard system (4.1).

Theorem 4.3. We assume that $\Omega \subset \mathbb{R}^{d}$ (with $d \in\{2,3\}$ ) is a bounded domain with Lipschitz boundary and that the potentials $F$ and $G$ satisfy the condition $(\mathrm{P} 2)$. Let $T>0$ and $m=\left(m_{1}, m_{2}\right) \in \mathbb{R}^{2}$ be arbitrary and let $\phi_{0} \in \mathcal{V}_{m}^{\kappa}$ be any initial datum having finite energy, i.e., $E\left(\phi_{0}\right)<\infty$. Then there exists a weak solution $\left(\phi, \mu, \mu_{\Gamma}\right)$ of the initial value problem (4.1) in the sense of Definition 4.1. This solution has the following additional properties:

$$
\begin{aligned}
\phi & \in C^{0,1 / 4}\left([0, T] ; L^{2}(\Omega)\right), \\
\left.\phi\right|_{\Gamma} & \in \begin{cases}C^{0,1 / 4}\left([0, T] ; L^{2}(\Gamma)\right), & \kappa>0, \\
C^{0,1 / 4}\left([0, T] ; H^{1}(\Gamma)^{*}\right), & \kappa=0\end{cases}
\end{aligned}
$$




\subsection{Implicit time discretization}

To prove Theorem 4.3, some preparation is necessary. The first step is to derive an implicit time discretization of the system (4.1). After that, we intend to show that the corresponding time-discrete solution converges to a weak solution of (4.1) in some suitable sense. This is a common approach in dealing with gradient flow equations that has already been used extensively in the literature (see, e.g., [4]).

To this end, let $N \in \mathbb{N}$ be arbitrary and let $\tau:=T / N$ denote the time step size. Without loss of generality, we assume that $\tau \leq 1$. Now, we define $\phi^{n}, n=0, \ldots, N$ recursively by the following construction: The 0 -th iterate is the initial datum, i.e., $\phi^{0}:=\phi_{0}$. If the $n$-th iterate $\phi^{n}$ is already constructed, we choose $\phi^{n+1}$ to be a minimizer of the functional

$$
J_{n}(\phi):=\frac{1}{2 \tau}\left\|\phi-\phi^{n}\right\|_{\mathcal{V}_{0}^{\kappa *}}^{2}+E(\phi)
$$

on the set $\mathcal{V}_{m}^{\kappa}$. Note that $J_{n}$ may attain the value $+\infty$. The existence of such a minimizer is guaranteed by Lemma 4.4 (that will be established in the next subsection). The idea behind this definition becomes clear when considering the first variation of the functional $J_{n}$ at the point $\phi^{n+1}$. As $\phi^{n+1}$ is a minimizer and since $F_{1}$ and $G_{1}$ are convex, we can proceed as in [21, Lem. 3.2] to conclude that

$$
\begin{gathered}
0=\left\langle\frac{\phi^{n+1}-\phi^{n}}{\tau}, \eta\right\rangle_{\mathcal{V}_{0}^{\kappa *}}+\int_{\Omega} \nabla \phi^{n+1} \cdot \nabla \eta \mathrm{d} x+\int_{\Omega} F^{\prime}\left(\phi^{n+1}\right) \eta \mathrm{d} x \\
+\int_{\Gamma} \kappa \nabla_{\Gamma} \phi^{n+1} \cdot \nabla_{\Gamma} \eta \mathrm{d} S(x)+\int_{\Gamma} G^{\prime}\left(\phi^{n+1}\right) \eta \mathrm{d} S(x)
\end{gathered}
$$

for all directions $\eta \in \mathcal{V}_{0}^{\kappa} \cap L^{\infty}(\Omega)$ with $\left.\eta\right|_{\Gamma} \in L^{\infty}(\Gamma)$. This can be interpreted as an implicit time discretization of the corresponding gradient flow equation (3.1). We set

$$
\stackrel{\circ}{\mu}^{n+1}:=(-\Delta)^{-1}\left(-\frac{\phi^{n+1}-\phi^{n}}{\tau}\right) \quad \text { and } \quad \stackrel{\circ}{\mu}_{\Gamma}^{n+1}:=\left(-\Delta_{\Gamma}\right)^{-1}\left(-\frac{\left.\phi^{n+1}\right|_{\Gamma}-\left.\phi^{n}\right|_{\Gamma}}{\tau}\right) .
$$

According to (P7), this means that $\stackrel{\leftrightarrow}{\mu}^{n+1} \in H^{1}(\Omega)$ and $\stackrel{\circ}{\mu}_{\Gamma}^{n+1} \in H^{1}(\Gamma)$ are a solution of the Poisson equations

$$
\begin{aligned}
-\Delta \mu & =-\frac{\phi^{n+1}-\phi^{n}}{\tau} \text { in } \Omega \text { with } \partial_{\mathbf{n}} \mu=0 \text { on } \Gamma, \\
-\Delta_{\Gamma} \mu_{\Gamma} & =-\frac{\phi^{n+1}-\phi^{n}}{\tau} \text { on } \Gamma
\end{aligned}
$$

with $\left\langle\dot{\mu}^{n+1}\right\rangle_{\Omega}=0$ and $\left\langle\dot{\mu}^{n+1}\right\rangle_{\Gamma}=0$. Hence, the functions $\phi^{n+1}, \phi^{n}, \stackrel{\circ}{\mu}^{n+1}$ and $\stackrel{\circ}{\mu}_{\Gamma}^{n+1}$ satisfy the equation

$$
\begin{aligned}
\int_{\Omega} \dot{\mu}^{n+1} \eta \mathrm{d} x+\int_{\Gamma} \dot{\mu}_{\Gamma}^{n+1} \eta \mathrm{d} S(x)= & \int_{\Omega} \nabla \phi^{n+1} \cdot \nabla \eta+F^{\prime}\left(\phi^{n+1}\right) \eta \mathrm{d} x \\
& +\int_{\Gamma} \kappa \nabla_{\Gamma} \phi^{n+1} \cdot \nabla_{\Gamma} \eta+G^{\prime}\left(\phi^{n+1}\right) \eta \mathrm{d} S(x)
\end{aligned}
$$

for all functions $\eta \in \mathcal{V}_{0}^{\kappa} \cap L^{\infty}(\Omega)$ with $\left.\eta\right|_{\Gamma} \in L^{\infty}(\Gamma)$ and all $n \in\{0, \ldots, N-1\}$. However, to obtain an approximate solution of the system (4.1), we need equation (4.9) to hold for all test functions $\eta \in \mathcal{V}^{\kappa} \cap L^{\infty}(\Omega)$ with $\left.\eta\right|_{\Gamma} \in L^{\infty}(\Gamma)$. The idea is to replace $\stackrel{\circ}{\mu}^{n+1}$ and $\stackrel{\circ}{\Gamma}_{\Gamma}^{n+1}$ by

$$
\mu^{n+1}:=\stackrel{\leftrightarrow}{\mu}^{n+1}+c^{n+1} \text { and } \mu_{\Gamma}^{n+1}:=\stackrel{\circ}{\mu}_{\Gamma}^{n+1}+c_{\Gamma}^{n+1},
$$

with constants $c^{n+1}, c_{\Gamma}^{n+1} \in \mathbb{R}$ that do not depend on $\eta$, since these functions $\mu^{n+1}$ and $\mu_{\Gamma}^{n+1}$ are still a solution of (4.7) and (4.8). We will now show that (4.9) holds true for all $\eta \in \mathcal{V}^{\kappa} \cap L^{\infty}(\Omega)$ with $\left.\eta\right|_{\Gamma} \in L^{\infty}(\Gamma)$ if the functions $\dot{\mu}^{n+1}$ and $\dot{\mu}_{\Gamma}^{n+1}$ are replaced by $\mu^{n+1}$ and $\mu_{\Gamma}^{n+1}$ with suitably chosen constants $c^{n+1}$ and $c_{\Gamma}^{n+1}$. Therefore, let $\eta \in \mathcal{V}^{\kappa} \cap L^{\infty}(\Omega)$ with $\left.\eta\right|_{\Gamma} \in L^{\infty}(\Gamma)$ be arbitrary. We define a new test function $\eta_{0}$ by

$$
\eta_{0}:=\eta-c_{1} \eta_{1}-c_{2} \eta_{2}
$$


where $c_{1}, c_{2} \in \mathbb{R}, \eta_{1} \equiv 1$ and $\eta_{2} \in C_{c}^{\infty}(\Omega)$ is an arbitrary nonnegative function that is not identically zero. Of course, this means that $\eta_{0} \in \mathcal{V}^{\kappa} \cap L^{\infty}(\Omega)$ with $\left.\eta\right|_{\Gamma} \in L^{\infty}(\Gamma)$. Choosing

$$
c_{1}:=\frac{1}{|\Gamma|} \int_{\Gamma} \eta \mathrm{d} S(x) \quad \text { and } \quad c_{2}:=\left(\int_{\Omega} \eta \mathrm{d} x-\frac{|\Omega|}{|\Gamma|} \int_{\Gamma} \eta \mathrm{d} S(x)\right) /\left(\int_{\Omega} \eta_{2} \mathrm{~d} x\right)
$$

we obtain that

$$
\begin{gathered}
\int_{\Gamma} \eta_{0} \mathrm{~d} S(x)=\int_{\Gamma} \eta \mathrm{d} S(x)-c_{1}|\Gamma|=0, \\
\int_{\Omega} \eta_{0} \mathrm{~d} x=\int_{\Omega} \eta \mathrm{d} x-c_{1}|\Omega|-c_{2} \int_{\Omega} \eta_{2} \mathrm{~d} x=0 .
\end{gathered}
$$

Thus, $\eta_{0}$ even lies in $\mathcal{V}_{0}^{\kappa} \cap L^{\infty}(\Omega)$ with $\left.\eta\right|_{\Gamma} \in L^{\infty}(\Gamma)$ and may thus be plugged into equation (4.9). This yields

$$
\begin{aligned}
\int_{\Omega} \nabla \phi^{n+1} \cdot & \nabla\left(\eta-c_{2} \eta_{2}\right)+F^{\prime}\left(\phi^{n+1}\right)\left(\eta-c_{1}-c_{2} \eta_{2}\right) \mathrm{d} x+\int_{\Gamma} \kappa \nabla_{\Gamma} \phi^{n+1} \cdot \nabla_{\Gamma} \eta \mathrm{d} S(x) \\
& +\int_{\Gamma} G^{\prime}\left(\phi^{n+1}\right)\left(\eta-c_{1}\right) \mathrm{d} S(x)=\int_{\Omega} \ddot{\mu}^{n+1}\left(\eta-c_{2} \eta\right) \mathrm{d} x+\int_{\Gamma} \stackrel{\mu}{\Gamma}_{\Gamma}^{n+1} \eta \mathrm{d} S(x)
\end{aligned}
$$

which is equivalent to

$$
\begin{aligned}
& \int_{\Omega} \nabla \phi^{n+1} \cdot \nabla \eta+F^{\prime}\left(\phi^{n+1}\right) \eta \mathrm{d} x+\int_{\Gamma} \kappa \nabla_{\Gamma} \phi^{n+1} \cdot \nabla_{\Gamma} \eta+G^{\prime}\left(\phi^{n+1}\right) \eta \mathrm{d} S(x) \\
& =\int_{\Omega} \grave{\mu}^{n+1} \eta \mathrm{d} x+\int_{\Gamma} \stackrel{\leftrightarrow}{\mu}_{\Gamma}^{n+1} \eta \mathrm{d} S(x)+c_{1} \int_{\Omega} F^{\prime}\left(\phi^{n+1}\right) \mathrm{d} x+c_{1} \int_{\Gamma} G^{\prime}\left(\phi^{n+1}\right) \mathrm{d} S(x) \\
& \quad-c_{2} \int_{\Omega} \stackrel{\mu}{\mu}^{n+1} \eta_{2} \mathrm{~d} x+c_{2} \int_{\Omega} \nabla \phi^{n+1} \cdot \nabla \eta_{2} \mathrm{~d} x+c_{2} \int_{\Omega} F^{\prime}\left(\phi^{n+1}\right) \eta_{2} \mathrm{~d} x .
\end{aligned}
$$

By the definition of $c_{1}$ and $c_{2}$ we obtain that

$$
\begin{gathered}
\int_{\Omega} \nabla \phi^{n+1} \cdot \nabla \eta+F^{\prime}\left(\phi^{n+1}\right) \eta \mathrm{d} x+\int_{\Gamma} \kappa \nabla_{\Gamma} \phi^{n+1} \cdot \nabla_{\Gamma} \eta+G^{\prime}\left(\phi^{n+1}\right) \eta \mathrm{d} S(x) \\
=\int_{\Omega}\left(\check{\mu}^{n+1}+c^{n+1}\right) \eta \mathrm{d} x+\int_{\Gamma}\left(\ddot{\mu}_{\Gamma}^{n+1}+c_{\Gamma}^{n+1}\right) \eta \mathrm{d} S(x)
\end{gathered}
$$

where the constants $c^{n+1}$ and $c_{\Gamma}^{n+1}$ are defined by

$$
\begin{aligned}
c^{n+1}:= & \left(\int_{\Omega}-\ddot{\mu}^{n+1} \eta_{2}+\nabla \phi^{n+1} \cdot \nabla \eta_{2}+F^{\prime}\left(\phi^{n+1}\right) \eta_{2} \mathrm{~d} x\right) /\left(\int_{\Omega} \eta_{2} \mathrm{~d} x\right), \\
c_{\Gamma}^{n+1}:= & \left(|\Omega| \int_{\Omega}-\stackrel{\leftrightarrow}{\mu}^{n+1} \eta_{2}+\nabla \phi^{n+1} \cdot \nabla \eta_{2}+F^{\prime}\left(\phi^{n+1}\right) \eta_{2} \mathrm{~d} x\right) /\left(|\Gamma| \int_{\Omega} \eta_{2} \mathrm{~d} x\right) \\
& +\frac{1}{|\Gamma|}\left(\int_{\Omega} F^{\prime}\left(\phi^{n+1}\right) \mathrm{d} x+\int_{\Gamma} G^{\prime}\left(\phi^{n+1}\right) \mathrm{d} S(x)\right) .
\end{aligned}
$$


Consequently the functions $\phi^{n+1}, \phi^{n}, \mu^{n+1}$ and $\mu_{\Gamma}^{n+1}$ are a solution of

$$
\begin{aligned}
& \left\langle\nabla \mu^{n+1}, \nabla \zeta\right\rangle_{L^{2}(\Omega)}=-\left\langle\frac{\phi^{n+1}-\phi^{n}}{\tau}, \zeta\right\rangle_{L^{2}(\Omega)} \quad \text { for all } \zeta \in H^{1}(\Omega), \\
& \left\langle\nabla_{\Gamma} \mu_{\Gamma}^{n+1}, \nabla_{\Gamma} \xi\right\rangle_{L^{2}(\Gamma)}=-\left\langle\frac{\phi^{n+1}-\phi^{n}}{\tau}, \xi\right\rangle_{L^{2}(\Gamma)} \quad \text { for all } \xi \in H^{1}(\Gamma), \\
& \int_{\Omega} \nabla \phi^{n+1} \cdot \nabla \eta+F^{\prime}\left(\phi^{n+1}\right) \eta \mathrm{d} x+\int_{\Gamma} \kappa \nabla_{\Gamma} \phi^{n+1} \cdot \nabla_{\Gamma} \eta+G^{\prime}\left(\phi^{n+1}\right) \eta \mathrm{d} S(x) \\
& \left.=\int_{\Omega} \mu^{n+1} \eta \mathrm{d} x+\int_{\Gamma} \mu_{\Gamma}^{n+1} \eta \mathrm{d} S(x) \quad \text { for all } \eta \in \mathcal{V}^{\kappa} \cap L^{\infty}(\Omega),\left.\eta\right|_{\Gamma} \in L^{\infty}(\Gamma)\right),
\end{aligned}
$$

which is an implicit time discretization of the system (4.1). This means that the triple $\left(\phi^{n}, \mu^{n}, \mu_{\Gamma}^{n}\right), n=$ $1, \ldots, N$ describes a time-discrete approximate solution.

In the following, $\left(\phi_{N}, \mu_{N}, \mu_{\Gamma, N}\right)$ will denote the piecewise constant extension of the approximate solution $\left(\phi^{n}, \mu^{n}, \mu_{\Gamma}^{n}\right)_{n=1, \ldots, N}$ on the interval $[0, T]$, i.e., for $n \in\{1, \ldots, N\}$ and $\left.\left.t \in\right](n-1) \tau, n \tau\right]$, we set

$$
\left(\phi_{N}, \mu_{N}, \mu_{\Gamma, N}\right)(\cdot, t):=\left(\phi_{N}^{n}, \mu_{N}^{n}, \mu_{\Gamma, N}^{n}\right):=\left(\phi^{n}, \mu^{n}, \mu_{\Gamma}^{n}\right) .
$$

Similarly, we define the piecewise linear extension $\left(\bar{\phi}_{N}, \bar{\mu}_{N}, \bar{\mu}_{\Gamma, N}\right)$ by

$$
\left(\bar{\phi}_{N}, \bar{\mu}_{N}, \bar{\mu}_{\Gamma, N}\right)(\cdot, t):=\alpha\left(\phi_{N}^{n}, \mu_{N}^{n}, \mu_{\Gamma, N}^{n}\right)+(1-\alpha)\left(\phi_{N}^{n-1}, \mu_{N}^{n-1}, \mu_{\Gamma, N}^{n-1}\right)
$$

for $n \in\{1, \ldots, N\}, \alpha \in[0,1]$ and $t=\alpha n \tau+(1-\alpha)(n-1) \tau$.

\subsection{The functional $J_{n}$ has a minimizer on $\mathcal{V}_{m}^{\kappa}$}

We now show existence of a time discrete solution using methods from calculus of variations.

Lemma 4.4. Let $N \in \mathbb{N}$ and $\tau>0$ as defined in Section 4.2 and let $n \in\{1, \ldots, N\}$ be arbitrary. Then the functional

$$
J_{n}(\phi):=\frac{1}{2 \tau}\left\|\phi-\phi^{n}\right\|_{\mathcal{V}_{0}^{\kappa *}}^{2}+E(\phi), \quad \phi \in \mathcal{V}_{m}^{\kappa}
$$

has a global minimizer $\bar{\phi} \in \mathcal{V}_{m}^{\kappa}$, i.e.,

$$
J_{n}(\bar{\phi}) \leq J_{n}(\phi), \quad \text { for all } \phi \in \mathcal{V}_{m}^{\kappa}
$$

Proof We can prove the existence of a minimizer by the direct method of calculus of variations. Obviously, $J$ is bounded from below by

$$
J_{n}(\phi) \geq \int_{\Omega} F(\phi) \mathrm{d} x+\int_{\Gamma} G(\phi) \mathrm{d} S \geq|\Omega| C_{F}+|\Gamma| C_{G}, \quad \text { for all } \phi \in \mathcal{V}_{m}^{\kappa}
$$

Thus, the infimum $M:=\inf _{\mathcal{V}_{m}^{\kappa}} J_{n}$ exists and therefore we can find a minimizing sequence $\left(\phi_{k}\right)_{k \in \mathbb{N}} \subset \mathcal{V}_{m}^{\kappa}$ with

$$
J_{n}\left(\phi_{k}\right) \rightarrow M, \quad k \rightarrow \infty \quad \text { and } \quad J_{n}\left(\phi_{k}\right) \leq M+1, \quad k \in \mathbb{N}
$$

From the definition of $J$, we conclude that

$$
\begin{aligned}
\frac{1}{2}\left\|\nabla \phi_{k}\right\|_{L^{2}(\Omega)}^{2}+\frac{\kappa}{2}\left\|\nabla_{\Gamma} \phi_{k}\right\|_{L^{2}(\Gamma)}^{2} & \leq J_{n}\left(\phi_{k}\right)-\int_{\Omega} F\left(\phi_{k}\right) \mathrm{d} x-\int_{\Gamma} G\left(\phi_{k}\right) \mathrm{d} S \\
& \leq M+1-C_{F}|\Omega|-C_{G}|\Omega|
\end{aligned}
$$


for all $k \in \mathbb{N}$. Since $\left\langle\phi_{k}\right\rangle_{\Omega}=m_{1}$ and $\left\langle\phi_{k}\right\rangle_{\Gamma}=m_{2}$ for all $k \in \mathbb{N}$, we can use Poincaré's inequality to infer that $\left(\phi_{k}\right)_{k \in \mathbb{N}}$ is bounded in the Hilbert space $\mathcal{V}^{\kappa}$. Hence, the Banach-Alaoglu theorem implies that there exists some function $\bar{\phi} \in \mathcal{V}^{\kappa}$ such that $\phi_{k} \rightarrow \bar{\phi}$ in $\mathcal{V}^{\kappa}$ after extraction of a subsequence. Then

$$
\left|\langle\bar{\phi}\rangle_{\Omega}-m_{1}\right|=\left|\langle\bar{\phi}\rangle_{\Omega}-\left\langle\phi_{k}\right\rangle_{\Omega}\right|=\frac{1}{|\Omega|}\left|\int_{\Omega} \bar{\phi}-\phi_{k} \mathrm{~d} x\right| \rightarrow 0, \quad k \rightarrow \infty
$$

since $\phi_{k} \rightarrow \bar{\phi}$ especially in $L^{2}(\Omega)$. This means that $\langle\bar{\phi}\rangle_{\Omega}=m_{1}$. The equality $\langle\bar{\phi}\rangle_{\Gamma}=m_{2}$ can be proved analogously due to the compact embedding $H^{1}(\Omega) \hookrightarrow L^{2}(\Gamma)$. As $H^{1}(\Omega)$ is compactly embedded in $L^{2}(\Omega)$ we obtain, after another subsequence extraction, that

$$
\phi_{k} \rightarrow \bar{\phi} \quad \text { in } L^{2}(\Omega) \quad \text { and } \quad \phi_{k} \rightarrow \bar{\phi} \quad \text { almost everywhere in } \Omega
$$

as $k \rightarrow \infty$. From the compact embedding $H^{1}(\Omega) \hookrightarrow L^{2}(\Gamma)$ we conclude that

$$
\phi_{k} \rightarrow \bar{\phi} \quad \text { in } L^{2}(\Gamma) \quad \text { and } \quad \phi_{k} \rightarrow \bar{\phi} \quad \text { almost everywhere in } \Gamma
$$

up to a subsequence. Obviously, all terms in the functional $J$ are convex in $\phi$ besides $\int_{\Omega} F_{2}(\phi) \mathrm{d} x$ and $\int_{\Gamma} G_{2}(\phi) \mathrm{d} S$. We know that $F_{2}\left(\phi_{k}\right) \rightarrow F_{2}(\bar{\phi})$ almost everywhere in $\Omega$ and $G_{2}\left(\phi_{k}\right) \rightarrow G_{2}(\bar{\phi})$ almost everywhere on $\Gamma$. Assumption (P2.4) implies that

$$
\left|F_{2}\left(\phi_{k}\right)\right| \leq C\left(1+\left|\phi_{k}\right|^{2}\right) \quad \text { in } \Omega \quad \text { and } \quad\left|G_{2}\left(\phi_{k}\right)\right| \leq C\left(1+\left|\phi_{k}\right|^{2}\right) \quad \text { on } \Gamma
$$

for some constant $C \geq 0$ depending only on $B_{F}$ and $B_{G}$. Hence Lebesgue's general convergence theorem (cf. [3, p. 60]) implies that

$$
\int_{\Omega} F_{2}\left(\phi_{k}(x)\right) \mathrm{d} x \rightarrow \int_{\Omega} F_{2}(\bar{\phi}(x)) \mathrm{d} x, \quad \int_{\Gamma} G_{2}\left(\phi_{k}(x)\right) \mathrm{d} S(x) \rightarrow \int_{\Gamma} G_{2}(\bar{\phi}(x)) \mathrm{d} S(x)
$$

as $k \rightarrow \infty$. Then, as the convex terms of $J$ are weakly lower semicontinuous, we obtain that

$$
J_{n}(\bar{\phi}) \leq \liminf _{k \rightarrow \infty} J_{n}\left(\phi_{k}\right)=M
$$

which directly yields $J_{n}(\bar{\phi})=M$ by the definition of $M$.

\subsection{Uniform bounds on the piecewise constant extension}

In this section the gradient flow structure will allow us to establish uniform a priori estimates.

Lemma 4.5. There exist nonnegative constants $C_{1}, \ldots, C_{5}$ that do not depend on $N, n$ or $\tau$ such that

$$
\begin{aligned}
& \left\|\phi_{N}\right\|_{L^{\infty}\left(0, T ; H^{1}(\Omega)\right)} \leq C_{1}, \\
& \begin{cases}\left\|\phi_{N}\right\|_{L^{\infty}\left(0, T ; H^{1 / 2}(\Gamma)\right)} \leq C_{2} & \text { if } \kappa=0, \\
\left\|\phi_{N}\right\|_{L^{\infty}\left(0, T ; H^{1}(\Gamma)\right)} \leq C_{3} & \text { if } \kappa>0,\end{cases} \\
& \left\|\mu_{N}\right\|_{L^{2}\left(0, T ; H^{1}(\Omega)\right)} \leq C_{4}, \\
& \left\|\mu_{\Gamma, N}\right\|_{L^{2}\left(0, T ; H^{1}(\Gamma)\right)} \leq C_{5}
\end{aligned}
$$

for all $N \in \mathbb{N}$.

Proof In the following, the letter $C$ will denote a generic nonnegative constant that does not depend on $n$, $\tau$ or $N$ and may change its value from line to line. First, as for any $n \in\{0, \ldots, N-1\}, \phi^{n+1}$ was chosen to be a minimizer of the functional $J_{n}$ on the set $\mathcal{V}_{m}^{\kappa}$, we obtain the a priori bound

$$
\frac{1}{2 \tau}\left\|\phi^{n+1}-\phi^{n}\right\|_{\mathcal{V}_{0}^{\kappa *}}^{2}+E\left(\phi^{n+1}\right)=J_{n}\left(\phi^{n+1}\right) \leq J_{n}\left(\phi^{n}\right)=E\left(\phi^{n}\right)
$$


for all $n \in\{0, \ldots, N-1\}$. It follows inductively that

$$
E\left(\phi^{n+1}\right) \leq E\left(\phi_{0}\right), \quad n \in\{0, \ldots, N-1\}
$$

From the definition of $E$ we conclude the uniform bound

$$
\begin{aligned}
& \frac{1}{2}\left\|\nabla \phi^{n+1}\right\|_{L^{2}(\Omega)}+\frac{\kappa}{2}\left\|\nabla_{\Gamma} \phi^{n+1}\right\|_{L^{2}(\Gamma)} \leq E\left(\phi_{0}\right)+C^{*}, \\
& \text { with } C^{*}:=-C_{F}|\Omega|-C_{G}|\Gamma|,
\end{aligned}
$$

for all $n \in\{0, \ldots, N-1\}$. Recall that $\left\langle\phi^{n+1}\right\rangle_{\Omega}=m_{1}$ and $\left\langle\phi^{n+1}\right\rangle_{\Gamma}=m_{2}$. Thus, by Poincaré's inequality,

$$
\begin{aligned}
\left\|\phi^{n+1}\right\|_{L^{2}(\Omega)} & \leq\left\|\left\langle\phi^{n+1}\right\rangle_{\Omega}\right\|_{L^{2}(\Omega)}+\left\|\phi^{n+1}-\left\langle\phi^{n+1}\right\rangle_{\Omega}\right\|_{L^{2}(\Omega)} \\
& \leq|\Omega|^{\frac{1}{2}} m_{1}+C\left\|\nabla \phi^{n+1}\right\|_{L^{2}(\Omega)} \leq C
\end{aligned}
$$

and, as the corresponding trace operator lies in $\mathcal{L}\left(H^{1}(\Omega) ; H^{1 / 2}(\Gamma)\right)$, we also have

$$
\left\|\phi^{n+1}\right\|_{H^{1 / 2}(\Gamma)} \leq C\left\|\phi^{n+1}\right\|_{H^{1}(\Omega)} .
$$

This means that $\phi_{N}$ is uniformly bounded in $L^{\infty}\left(0, T ; H^{1}(\Omega)\right)$ and its trace $\left.\phi_{N}\right|_{\Gamma}$ is uniformly bounded in $L^{\infty}\left(0, T ; H^{1 / 2}(\Gamma)\right)$. If $\kappa>0$, the trace $\left.\phi_{N}\right|_{\Gamma}$ is even uniformly bounded in $L^{\infty}\left(0, T ; H^{1}(\Gamma)\right)$ according to (4.19). Recalling the definition of $\phi_{N}$, this proves (4.13) and (4.14).

Now, let $\eta \in C_{c}^{\infty}(\Omega ;[0,1])$ be any function that is not identically zero. Then equation (4.10c) implies for all such $\eta$ that

$$
\int_{\Omega} \nabla \phi^{n+1} \cdot \nabla \eta \mathrm{d} x+\int_{\Omega} F^{\prime}\left(\phi^{n+1}\right) \eta \mathrm{d} x=\int_{\Omega} \mu^{n+1} \eta \mathrm{d} x .
$$

Due to (P2.3) with $\delta=1$, the second summand on the left-hand side can be bounded by

$$
\begin{aligned}
\left|\int_{\Omega} F^{\prime}\left(\phi^{n+1}\right) \eta \mathrm{d} x\right| & \leq \int_{\Omega} F_{1}\left(\phi^{n+1}\right) \mathrm{d} x+B_{F}|\Omega|^{1 / 2}\left\|\phi^{n+1}\right\|_{L^{2}(\Omega)}+\left(A_{F}^{1}+B_{F}\right)|\Omega| \\
& \leq E\left(\phi_{0}\right)+C^{*}+B_{F}|\Omega|^{1 / 2}\left\|\phi^{n+1}\right\|_{L^{2}(\Omega)}+\left(A_{F}^{1}+B_{F}\right)|\Omega| \leq C .
\end{aligned}
$$

Hence there exists some constant $C(\eta) \geq 0$ such that

$$
\left|\int_{\Omega} \mu^{n+1} \eta \mathrm{d} x\right| \leq C(\eta)
$$

Let us now define the set

$$
\mathcal{M}_{\eta}:=\left\{v \in H^{1}(\Omega)|| \int_{\Omega} v \eta \mathrm{d} x \mid \leq C(\eta)\right\}
$$

This set is obviously a non-empty, closed and convex subset of $H^{1}(\Omega)$. This means that the generalized Poincaré inequality (Lemma 6.1) can be applied to this set with $u_{0}=0$ and

$$
C_{0}:=\frac{C(\eta)}{\left|\int_{\Omega} \eta \mathrm{d} x\right|}
$$

because then all numbers $\xi \in \mathbb{R}$ with $\xi \chi_{\Omega} \in \mathcal{M}_{\eta}$ satisfy

$$
|\xi| \leq \frac{\left|\int_{\Omega} \xi \eta \mathrm{d} x\right|}{\left|\int_{\Omega} \eta \mathrm{d} x\right|} \leq C_{0}
$$


We obtain that

$$
\|\mu\|_{L^{2}(\Omega)} \leq C\left(1+\|\nabla \mu\|_{L^{2}(\Omega)}\right), \quad \text { for all } \mu \in \mathcal{M}_{\eta} .
$$

We know from estimate (4.23) that $\mu^{n+1} \in \mathcal{M}_{\eta}$ and thus

$$
\left\|\mu^{n+1}\right\|_{L^{2}(\Omega)} \leq C\left(1+\left\|\nabla \mu^{n+1}\right\|_{L^{2}(\Omega)}\right), \quad \text { for all } n \in\{0, \ldots, N-1\} .
$$

To establish a uniform bound on $\mu_{N}$, let $n \in\{1, \ldots, N\}$ be arbitrary and set $t:=n \tau$. Then, for any $\left.\left.s \in\right] t-\tau, t\right]$, we have

$$
\phi_{N}(s)=\phi_{N}(t)=\phi_{N}^{n}, \quad \mu_{N}(s)=\mu_{N}(t)=\mu_{N}^{n} \quad \text { and } \quad \mu_{\Gamma, N}(s)=\mu_{\Gamma, N}(t)=\mu_{\Gamma, N}^{n} .
$$

Using the a priori estimate (4.17) and the definition of $\mu_{N}$ and $\mu_{\Gamma, N}$, we obtain that

$$
\begin{aligned}
& E\left(\phi_{N}(t)\right)+\frac{1}{2} \int_{t-\tau}^{t}\left\|\nabla \mu_{N}(s)\right\|_{L^{2}(\Omega)}^{2}+\left\|\nabla_{\Gamma} \mu_{\Gamma, N}(s)\right\|_{L^{2}(\Gamma)}^{2} \mathrm{~d} s \\
& =E\left(\phi_{N}(t)\right)+\frac{1}{2} \int_{t-\tau}^{t} \frac{1}{\tau^{2}}\left\|\phi_{N}(s)-\phi_{N}(s-\tau)\right\|_{\mathcal{V}_{0}^{\kappa *}}^{2} \mathrm{~d} s \\
& =E\left(\phi_{N}(t)\right)+\frac{1}{2} \int_{t-\tau}^{t} \frac{1}{\tau^{2}}\left\|\phi_{N}(t)-\phi_{N}(t-\tau)\right\|_{\mathcal{V}_{0}^{\kappa *}}^{2} \mathrm{~d} s \\
& =E\left(\phi_{N}(t)\right)+\frac{1}{2 \tau}\left\|\phi_{N}(t)-\phi_{N}(t-\tau)\right\|_{\mathcal{V}_{0}^{\kappa *}}^{2} \\
& \quad \leq E\left(\phi_{N}(t-\tau)\right) .
\end{aligned}
$$

It follows inductively that

$$
E\left(\phi_{N}(t)\right)+\frac{1}{2} \int_{0}^{t}\left\|\nabla \mu_{N}(s)\right\|_{L^{2}(\Omega)}^{2}+\left\|\nabla_{\Gamma} \mu_{\Gamma, N}(s)\right\|_{L^{2}(\Gamma)}^{2} \mathrm{~d} s \leq E\left(\phi_{0}\right)
$$

and in particular,

$$
\int_{0}^{T}\left\|\nabla \mu_{N}(s)\right\|_{L^{2}(\Omega)}^{2}+\left\|\nabla \mu_{\Gamma, N}(s)\right\|_{L^{2}(\Gamma)}^{2} \mathrm{~d} s \leq 2\left(E\left(\phi_{0}\right)+C^{*}\right) \leq C .
$$

Inequality (4.24) directly yields

$$
\left.\left.\left\|\mu_{N}(s)\right\|_{L^{2}(\Omega)} \leq C\left(1+\left\|\nabla \mu_{N}(s)\right\|_{L^{2}(\Omega)}\right), \quad s \in\right] 0, T\right]
$$

and thus we can conclude that $\mu_{N}$ is uniformly bounded in $L^{2}\left(0, T ; H^{1}(\Omega)\right)$ which means that (4.15) is established.

Testing equation (4.10c) with $\eta \equiv 1$ gives

$$
\int_{\Gamma} \mu_{\Gamma, N}(t) \mathrm{d} S=\int_{\Gamma} G^{\prime}\left(\phi_{N}(t)\right) \mathrm{d} S+\int_{\Omega} F^{\prime}\left(\phi_{N}(t)\right) \mathrm{d} x-\int_{\Omega} \mu_{N}(t) \mathrm{d} x,
$$

for all $t \in[0, T]$. From (P2.3) with $\delta=1$ and estimate (4.25) we deduce that

$$
\left|\int_{\Gamma} G^{\prime}\left(\phi_{N}(t)\right) \mathrm{d} S\right| \leq \int_{\Gamma} G_{1}\left(\phi_{N}(t)\right) \mathrm{d} x+B_{G}|\Gamma|^{1 / 2}\left\|\phi_{N}(t)\right\|_{L^{2}(\Gamma)}+\left(A_{G}^{1}+B_{G}\right)\left|\Gamma_{T}\right|
$$




$$
\leq E\left(\phi_{0}\right)+C^{*}+B_{G}|\Gamma|^{1 / 2}\left\|\phi_{N}\right\|_{L^{\infty}\left(0, T ; L^{2}(\Gamma)\right)}+\left(A_{G}^{1}+B_{G}\right)\left|\Gamma_{T}\right|
$$

for all $t \in[0, T]$. Recall that $\phi_{N}$ is uniformly bounded in $L^{\infty}\left(0, T ; L^{2}(\Gamma)\right)$. The second integral on the right-hand side of (4.28) can be bounded analogously and we obtain that

$$
\left|\int_{\Gamma} G^{\prime}\left(\phi_{N}(t)\right) \mathrm{d} S\right| \leq C \quad \text { and }\left|\int_{\Omega} F^{\prime}\left(\phi_{N}(t)\right) \mathrm{d} x\right| \leq C, \quad t \in[0, T] .
$$

Consequently,

$$
\left|\int_{\Gamma} \mu_{\Gamma, N}(t) \mathrm{d} S\right| \leq C+\int_{\Omega}\left|\mu_{N}(t)\right| \mathrm{d} x, \quad t \in[0, T]
$$

and thus, since $\mu_{N}$ is uniformly bounded in $L^{2}\left(0, T ; L^{2}(\Omega)\right)$,

$$
\begin{aligned}
\int_{0}^{T}\left\|\left\langle\mu_{\Gamma, N}(t)\right\rangle_{\Gamma}\right\|_{L^{2}(\Gamma)}^{2} \mathrm{~d} t & \leq C+C \int_{0}^{T}\left(\int_{\Omega}\left|\mu_{N}(t)\right| \mathrm{d} x\right)^{2} \mathrm{~d} t \\
& \leq C+C\left\|\mu_{N}\right\|_{L^{2}\left(0, T ; L^{2}(\Omega)\right)}^{2} \leq C .
\end{aligned}
$$

Then, by Poincaré's inequality,

$$
\begin{aligned}
& \int_{0}^{T}\left\|\mu_{\Gamma, N}(t)\right\|_{L^{2}(\Gamma)}^{2} \mathrm{~d} t \\
& \quad \leq C \int_{0}^{T}\left\|\left\langle\mu_{\Gamma, N}(t)\right\rangle_{\Gamma}\right\|_{L^{2}(\Gamma)}^{2} \mathrm{~d} t+C \int_{0}^{T}\left\|\mu_{\Gamma, N}(t)-\left\langle\mu_{\Gamma, N}(t)\right\rangle_{\Gamma}\right\|_{L^{2}(\Gamma)}^{2} \mathrm{~d} t \\
& \quad \leq C+C \int_{0}^{T}\left\|\nabla_{\Gamma} \mu_{\Gamma, N}(t)\right\|_{L^{2}(\Gamma)}^{2} \mathrm{~d} t
\end{aligned}
$$

for all $t \in[0, T]$. Finally, (4.26) implies that $\mu_{\Gamma, N}$ is uniformly bounded in the space $L^{2}\left(0, T ; H^{1}(\Gamma)\right)$ which proves assertion (4.16).

\subsection{Hölder estimates for the piecewise continuous extension}

Via interpolation type arguments we can show Hölder continuity in time.

Lemma 4.6. There exists some constant $C>0$ that does not depend on $N$ such that for all $t_{1}, t_{2} \in[0, T]$,

$$
\begin{aligned}
\left\|\bar{\phi}_{N}\left(t_{1}\right)-\bar{\phi}_{N}\left(t_{2}\right)\right\|_{L^{2}(\Omega)} & \leq C\left|t_{1}-t_{2}\right|^{1 / 4}, & & \\
\left\|\bar{\phi}_{N}\left(t_{1}\right)-\bar{\phi}_{N}\left(t_{2}\right)\right\|_{L^{2}(\Gamma)} & \leq C\left|t_{1}-t_{2}\right|^{1 / 4}, & & \text { if } \kappa>0, \\
\left\|\bar{\phi}_{N}\left(t_{1}\right)-\bar{\phi}_{N}\left(t_{2}\right)\right\|_{H^{1}(\Gamma)^{*}} & \leq C\left|t_{1}-t_{2}\right|^{1 / 4}, & & \text { if } \kappa=0 .
\end{aligned}
$$

Proof Let $t_{1}, t_{2} \in[0, T]$ be arbitrary. Without loss of generality, we assume that $t_{1}<t_{2}$. Since $\bar{\phi}_{N}$ is piecewise linear in time, it is weakly differentiable with respect to $t$ and we can rewrite the equations (4.10a) and $(4.10 \mathrm{~b})$ as

$$
\int_{\Omega} \partial_{t} \bar{\phi}_{N}(t) \zeta \mathrm{d} x=-\int_{\Omega} \nabla \mu_{N}(t) \cdot \nabla \zeta \mathrm{d} x \quad \text { for all } \zeta \in H^{1}(\Omega)
$$




$$
\int_{\Gamma} \partial_{t} \bar{\phi}_{N}(t) \xi \mathrm{d} S=-\int_{\Gamma} \nabla_{\Gamma} \mu_{\Gamma, N}(t) \cdot \nabla_{\Gamma} \xi \mathrm{d} S \quad \text { for all } \xi \in H^{1}(\Gamma)
$$

for all $t \in[0, T]$. Choosing $\zeta:=\bar{\phi}_{N}\left(t_{2}\right)-\bar{\phi}_{N}\left(t_{1}\right)$ and integrating with respect to $t$ from $t_{1}$ to $t_{2}$ we obtain that

$$
\begin{gathered}
\left\|\bar{\phi}_{N}\left(t_{2}\right)-\bar{\phi}_{N}\left(t_{1}\right)\right\|_{L^{2}(\Omega)}^{2}=-\int_{t_{1}}^{t_{2}} \int_{\Omega} \nabla \mu_{N}(t) \cdot\left(\nabla \bar{\phi}_{N}\left(t_{2}\right)-\nabla \bar{\phi}_{N}\left(t_{1}\right)\right) \mathrm{d} x \mathrm{~d} t \\
\leq 2\left\|\phi_{N}\right\|_{L^{\infty}\left(0, T ; H^{1}(\Omega)\right)}\left\|\mu_{N}\right\|_{L^{2}\left(0, T ; H^{1}(\Omega)\right)}\left|t_{2}-t_{1}\right|^{1 / 2} \leq C\left|t_{2}-t_{1}\right|^{1 / 2},
\end{gathered}
$$

which proves assertion (4.30). Assertion (4.31) can be proved analogously by choosing the test function $\xi:=\left.\bar{\phi}_{N}\right|_{\Gamma}\left(t_{2}\right)-\left.\bar{\phi}_{N}\right|_{\Gamma}\left(t_{1}\right)$. However, if $\kappa=0$, we have to proceed differently since $\left(\left.\bar{\phi}_{N}\right|_{\Gamma}\right)_{N \in \mathbb{N}}$ is merely bounded in $L^{\infty}\left(0, T ; L^{2}(\Gamma)\right)$. As $\left\langle\bar{\phi}\left(t_{2}\right)-\bar{\phi}\left(t_{1}\right)\right\rangle_{\Gamma}=0$ it holds that

$$
\begin{aligned}
& \left\|\bar{\phi}\left(t_{2}\right)-\bar{\phi}\left(t_{1}\right)\right\|_{H^{1}(\Gamma)^{*}}^{2} \\
& \quad=\int_{\Gamma} \nabla_{\Gamma}\left(-\Delta_{\Gamma}\right)^{-1}\left(\bar{\phi}_{N}\left(t_{2}\right)-\bar{\phi}_{N}\left(t_{1}\right)\right) \cdot \nabla_{\Gamma}\left(-\Delta_{\Gamma}\right)^{-1}\left(\bar{\phi}_{N}\left(t_{2}\right)-\bar{\phi}_{N}\left(t_{1}\right)\right) \mathrm{d} S \\
& \quad=\int_{\Gamma}\left(\bar{\phi}_{N}\left(t_{2}\right)-\bar{\phi}_{N}\left(t_{1}\right)\right)\left(-\Delta_{\Gamma}\right)^{-1}\left(\bar{\phi}_{N}\left(t_{2}\right)-\bar{\phi}_{N}\left(t_{1}\right)\right) \mathrm{d} S .
\end{aligned}
$$

Hence, choosing $\xi:=\left(-\Delta_{\Gamma}\right)^{-1}\left(\bar{\phi}_{N}\left(t_{2}\right)-\bar{\phi}_{N}\left(t_{1}\right)\right) \in H^{1}(\Gamma)$ yields

$$
\begin{aligned}
& \left\|\bar{\phi}\left(t_{2}\right)-\bar{\phi}\left(t_{1}\right)\right\|_{H^{1}(\Gamma)^{*}}^{2}=\int_{\Gamma}\left(\bar{\phi}_{N}\left(t_{2}\right)-\bar{\phi}_{N}\left(t_{1}\right)\right) \xi \mathrm{d} S=-\int_{t_{1}}^{t_{2}} \int_{\Gamma} \nabla_{\Gamma} \mu_{\Gamma, N}(t) \cdot \nabla_{\Gamma} \xi \mathrm{d} S \mathrm{~d} t \\
& \quad=\int_{t_{1}}^{t_{2}} \int_{\Gamma} \mu_{\Gamma, N}(t)\left(\bar{\phi}_{N}\left(t_{2}\right)-\bar{\phi}_{N}\left(t_{1}\right)\right) \mathrm{d} S \mathrm{~d} t \\
& \quad \leq 2\left\|\mu_{\Gamma, N}\right\|_{L^{2}\left(0, T ; L^{2}(\Gamma)\right)}\left\|\bar{\phi}_{N}\right\|_{L^{\infty}\left(0, T ; L^{2}(\Gamma)\right)}\left|t_{2}-t_{1}\right|^{1 / 2},
\end{aligned}
$$

which proves assertion (4.32).

\subsection{Convergence of the approximate solution}

In this section we will use the apriori estimates and compactness arguments to show convergence of the time discrete solutions.

Lemma 4.7. There exist functions

$$
\begin{aligned}
& \phi \in L^{\infty}\left(0, T ; H^{1}(\Omega)\right) \cap C^{0, \frac{1}{4}}\left([0, T] ; L^{2}(\Omega)\right) \\
& \psi \in \begin{cases}L^{\infty}\left(0, T ; H^{1}(\Gamma)\right) \cap C^{0, \frac{1}{4}}\left([0, T] ; L^{2}(\Gamma)\right), & \kappa>0, \\
L^{\infty}\left(0, T ; H^{1 / 2}(\Gamma)\right) \cap C^{0, \frac{1}{4}}\left([0, T] ; H^{1}(\Gamma)^{*}\right) \cap C\left([0, T] ; L^{2}(\Gamma)\right), & \kappa=0,\end{cases} \\
& \mu \in L^{2}\left(0, T ; H^{1}(\Omega)\right), \\
& \mu_{\Gamma} \in L^{2}\left(0, T ; H^{1}(\Gamma)\right)
\end{aligned}
$$

such that for any $\gamma \in] 0, \frac{1}{4}[$,

$$
\begin{array}{ll}
\phi_{N} \stackrel{*}{\rightarrow} \phi & \text { in } L^{\infty}\left(0, T ; H^{1}(\Omega)\right), \\
\bar{\phi}_{N} \rightarrow \phi & \text { in } C^{0, \gamma}\left([0, T] ; L^{2}(\Omega)\right),
\end{array}
$$




$$
\begin{array}{cl}
\phi_{N} \rightarrow \phi & \text { in } L^{\infty}\left(0, T ; L^{2}(\Omega)\right), \\
\phi_{N} \rightarrow \phi & \text { almost everywhere in } \Omega_{T}, \\
\left.\phi_{N}\right|_{\Gamma} \stackrel{*}{\rightarrow} \psi & \text { in } \begin{cases}L^{\infty}\left(0, T ; H^{1}(\Gamma)\right), & \kappa>0, \\
L^{\infty}\left(0, T ; H^{1 / 2}(\Gamma)\right), & \kappa=0,\end{cases} \\
\left.\bar{\phi}_{N}\right|_{\Gamma} \rightarrow \psi & \text { in } \begin{cases}C^{0, \gamma}\left([0, T] ; L^{2}(\Gamma)\right), & \kappa>0, \\
C^{0, \gamma}\left([0, T] ; H^{1}(\Gamma)^{*}\right), & \kappa=0,\end{cases} \\
\left.\phi_{N}\right|_{\Gamma} \rightarrow \psi & \text { in } \begin{cases}L^{\infty}\left(0, T ; L^{2}(\Gamma)\right), & \kappa>0, \\
L^{\infty}\left(0, T ; H^{1}(\Gamma)^{*}\right), & \kappa=0,\end{cases} \\
\left.\phi_{N}\right|_{\Gamma} \rightarrow \psi & \text { almost everywhere on } \Gamma_{T}, \\
\left.\phi_{N}\right|_{\Gamma} \rightarrow \psi & \text { in } C\left([0, T] ; L^{2}(\Gamma)\right), \\
\mu_{N} \rightarrow \mu & \text { in } L^{2}\left(0, T ; H^{1}(\Omega)\right), \\
\mu_{\Gamma, N} \rightarrow \mu_{\Gamma} & \text { in } L^{2}\left(0, T ; H^{1}(\Gamma)\right)
\end{array}
$$

up to a subsequence as $N \rightarrow \infty$. Moreover, it holds that $\psi=\left.\phi\right|_{\Gamma}$ almost everywhere on $\Gamma_{T}$.

Proof Due to the bounds (4.13)-(4.16) that have been established in Lemma 4.5, we can conclude from the Banach-Alaoglu theorem that there exist functions

$$
\begin{array}{ll}
\phi \in L^{\infty}\left(0, T ; H^{1}(\Omega)\right), & \psi \in \begin{cases}L^{\infty}\left(0, T ; H^{1}(\Gamma)\right), & \kappa>0, \\
L^{\infty}\left(0, T ; H^{1 / 2}(\Gamma)\right), & \kappa=0,\end{cases} \\
\mu \in L^{2}\left(0, T ; H^{1}(\Omega)\right), & \mu_{\Gamma} \in L^{2}\left(0, T ; H^{1}(\Gamma)\right)
\end{array}
$$

such that, after extraction of a subsequence,

$$
\begin{aligned}
& \phi_{N} \stackrel{*}{\rightarrow} \phi \quad \text { in } \quad L^{\infty}\left(0, T ; H^{1}(\Omega)\right),\left.\quad \phi_{N}\right|_{\Gamma} \stackrel{*}{*} \psi \quad \text { in } \quad \begin{array}{ll}
L^{\infty}\left(0, T ; H^{1}(\Gamma)\right), & \kappa>0, \\
L^{\infty}\left(0, T ; H^{1 / 2}(\Gamma)\right), & \kappa=0,
\end{array} \\
& \mu_{N} \rightarrow \mu \quad \text { in } \quad L^{2}\left(0, T ; H^{1}(\Omega)\right), \\
& \mu_{\Gamma, N} \rightarrow \mu_{\Gamma} \quad \text { in } \quad L^{2}\left(0, T ; H^{1}(\Gamma)\right) .
\end{aligned}
$$

Hence, the assertions (4.35), (4.39), (4.44) and (4.45) are established.

Recall that $\bar{\phi}_{N}$ lies in $C\left([0, T] ; L^{2}(\Omega)\right)$ and is bounded uniformly in $L^{\infty}\left(0, T ; H^{1}(\Omega)\right)$ by

$$
\left\|\bar{\phi}_{N}\right\|_{L^{\infty}\left(0, T ; H^{1}(\Omega)\right)} \leq\left\|\phi_{N}\right\|_{L^{\infty}\left(0, T ; H^{1}(\Omega)\right)} \leq C_{1}
$$

according to (4.13) for all $N \in \mathbb{N}$. Since the embedding from $H^{1}(\Omega)$ to $L^{2}(\Omega)$ is compact, we can use the equicontinuity of $\left(\bar{\phi}_{N}\right)_{N \in \mathbb{N}}$ (which follows directly from (4.30)) to apply the theorem of Arzelà-Ascoli for functions with values in a Banach space (see J. Simon [32, Lem. 1]). The theorem implies that

$$
\bar{\phi}_{N} \rightarrow \phi \quad \text { in } C\left([0, T] ; L^{2}(\Omega)\right) .
$$

Using (4.30) one can easily show that $\phi \in C^{0, \frac{1}{4}}\left([0, T] ; L^{2}(\Omega)\right)$. For any $\left.\gamma \in\right] 0, \frac{1}{4}[$, we obtain by interpolation that

$$
\|\cdot\|_{C^{0, \gamma}\left([0, T] ; L^{2}(\Omega)\right)} \leq C\|\cdot\|_{C^{0,1 / 4}\left([0, T] ; L^{2}(\Omega)\right)}^{4 \gamma}\|\cdot\|_{C\left([0, T] ; L^{2}(\Omega)\right)}^{1-4 \gamma} \cdot
$$

Hence, it also holds that

$$
\bar{\phi}_{N} \rightarrow \phi \quad \text { in } C^{0, \gamma}\left([0, T] ; L^{2}(\Omega)\right)
$$


for every $\gamma \in] 0, \frac{1}{4}[$. This proves (4.36). Assertion (4.40) can be established analogously; in the case $\kappa=0$ we have to use the compact embedding $L^{2}(\Gamma) \cong L^{2}(\Gamma)^{*} \hookrightarrow H^{1}(\Gamma)^{*}$ instead.

For any $t \in[0, T]$ we can choose $n \in\{1, \ldots, N\}$ and $\alpha \in[0,1]$ such that $t=\alpha n \tau+(1-\alpha)(n-1) \tau$. We have

$$
\begin{aligned}
& \left\|\bar{\phi}_{N}(t)-\phi_{N}(t)\right\|_{L^{2}(\Omega)} \leq\left\|\alpha \phi^{n}+(1-\alpha) \phi_{N}^{n-1}(t)-\phi_{N}^{n}(t)\right\|_{L^{2}(\Omega)} \\
& \quad=(1-\alpha)\left\|\phi_{N}^{n}(t)-\phi_{N}^{n-1}(t)\right\|_{L^{2}(\Omega)} \leq C \tau^{1 / 4}
\end{aligned}
$$

which tends to zero as $N$ tends to infinity. Proceeding similarly, we obtain that

$$
\begin{aligned}
\left\|\bar{\phi}_{N}(t)-\phi_{N}(t)\right\|_{L^{2}(\Gamma)} & \leq C \tau^{1 / 4} \underset{N \rightarrow \infty}{\longrightarrow} 0, & & \text { if } \kappa>0, \\
\left\|\bar{\phi}_{N}(t)-\phi_{N}(t)\right\|_{H^{1}(\Gamma)^{*}} & \leq C \tau^{1 / 4} \underset{N \rightarrow \infty}{\longrightarrow} 0, & & \text { if } \kappa=0 .
\end{aligned}
$$

Together with (4.36) and (4.40) this implies (4.37) and (4.41). In particular, this means that $\phi_{N} \rightarrow \phi$ in $L^{2}\left(\Omega_{T}\right)$ and $\left.\left.\phi_{N}\right|_{\Gamma} \rightarrow \phi\right|_{\Gamma}$ in $L^{2}\left(\Gamma_{T}\right)$ if $\kappa>0$. Therefore we can extract a subsequence that converges almost everywhere. This proves (4.38) and (4.42) in the case $\kappa>0$.

By integration, (4.34) implies that

$$
\int_{\Gamma_{T}} \partial_{t} \bar{\phi}_{N} \xi \mathrm{d} S(x) \mathrm{d} t=-\int_{\Gamma_{T}} \nabla_{\Gamma} \mu_{\Gamma, N} \cdot \nabla_{\Gamma} \xi \mathrm{d}(x, t)
$$

for all functions $\xi \in L^{2}\left(0, T ; H^{1}(\Gamma)\right)$ with $\partial_{t} \xi \in L^{2}\left(\Gamma_{T}\right)$ and consequently

$$
\partial_{t} \bar{\phi}_{N}=\Delta_{\Gamma} \mu_{\Gamma, N} \quad \text { in } L^{2}\left(0, T ; H^{1}(\Gamma)^{*}\right)
$$

for all $N \in \mathbb{N}$. This means that $\partial_{t} \phi_{N}$ is uniformly bounded by

$$
\left\|\partial_{t} \bar{\phi}_{N}\right\|_{L^{2}\left(0, T ; H^{1}(\Gamma)^{*}\right)}=\left\|\Delta_{\Gamma} \mu_{\Gamma, N}\right\|_{L^{2}\left(0, T ; H^{1}(\Gamma)^{*}\right)}=\left\|\nabla_{\Gamma} \mu_{\Gamma, N}\right\|_{L^{2}\left(0, T ; L^{2}(\Gamma)\right)} \leq C_{5} .
$$

according to (4.16) and the definition of $\mu_{\Gamma, N}$. Hence, the sequence $\left(\bar{\phi}_{N}\right)_{N \in \mathbb{N}}$ is bounded in $L^{\infty}\left(0, T ; H^{1 / 2}(\Gamma)\right) \cap$ $H^{1}\left(0, T ; H^{1}(\Gamma)^{*}\right)$. Since $H^{1 / 2}(\Gamma)$ is compactly embedded in $L^{2}(\Gamma)$ and $L^{2}(\Gamma) \cong L^{2}(\Gamma)^{*}$ is continuously embedded in $H^{1}(\Gamma)^{*}$ we can use the Aubin-Lions lemma (cf. J. Simon[32, Cor. 5]) to conclude that

$$
\left.\bar{\phi}_{N}\right|_{\Gamma} \rightarrow \psi \text { in } C\left([0, T] ; L^{2}(\Gamma)\right) .
$$

up to a subsequence which is (4.43). Then assertion (4.42) in the case $\kappa=0$ immediately follows after another subsequence extraction.

Similarly, we can use (4.33) to conclude that

$$
\partial_{t} \bar{\phi}_{N}=\Delta \mu_{\Gamma, N} \quad \text { in } L^{2}\left(0, T ; H^{1}(\Omega)^{*}\right)
$$

with

$$
\left\|\partial_{t} \bar{\phi}_{N}\right\|_{L^{2}\left(0, T ; H^{1}(\Omega)^{*}\right)}=\left\|\Delta_{\Gamma} \mu_{\Gamma, N}\right\|_{L^{2}\left(0, T ; H^{1}(\Omega)^{*}\right)}=\left\|\nabla_{\Gamma} \mu_{\Gamma, N}\right\|_{L^{2}\left(0, T ; L^{2}(\Omega)\right)} \leq C_{4}
$$

and therefore, $\left(\bar{\phi}_{N}\right)_{N \in \mathbb{N}}$ is bounded in $L^{\infty}\left(0, T ; H^{1}(\Omega)\right) \cap H^{1}\left(0, T ; H^{1}(\Omega)^{*}\right)$. It holds that

$$
\left.H^{1}(\Omega) \hookrightarrow H^{s}(\Omega) \hookrightarrow L^{2}(\Omega) \cong L^{2}(\Omega)^{*} \hookrightarrow H^{1}(\Omega)^{*} \quad \text { for any } s \in\right] 0,1[
$$

where all embeddings are continuous and at least the first embedding is compact. Now, the Aubin-Lions lemma (cf. J. Simon[32, Cor. 5]) implies that $\left.\phi_{N}\right|_{\Gamma} \rightarrow \phi$ in $C\left([0, T] ; H^{s}(\Omega)\right)$ for any fixed $\left.s \in\right] 0,1[$ up to a subsequence. Choosing $s>\frac{1}{2}$, we can conclude from the continuity of the trace operator (see (P5)) that

$$
\left.\left.\bar{\phi}_{N}\right|_{\Gamma} \rightarrow \phi\right|_{\Gamma} \text { in } C\left([0, T] ; L^{2}(\Gamma)\right) .
$$

after subsequence extraction. This finally proves $\left.\phi\right|_{\Gamma}=\psi$. 


\subsection{Proof of the existence theorem}

We can finally prove our main result Theorem 4.3 by showing that the limit $\left(\phi, \mu, \mu_{\Gamma}\right)$ from Lemma 4.7 is a weak solution of the Cahn-Hilliard equation (4.1) in the sense of Definition 4.1.

Proof of Theorem 4.3. First note that $\left(\phi, \mu, \mu_{\Gamma}\right)$ has the desired regularity according to Lemma 4.7. This means that item (i) of Definition 4.1 is already satisfied. To verify (ii), we need to pass the limit in the discretized system (4.10a)-(4.10c) using the convergence results that were established in Lemma 4.7. First, the equations (4.33) and (4.34) imply that

$$
\int_{\Omega_{T}}\left(\bar{\phi}_{N}-\phi_{0}\right) \partial_{t} \zeta \mathrm{d}(x, t)=\int_{\Omega_{T}} \nabla \mu_{N} \cdot \nabla \zeta \mathrm{d}(x, t)
$$

for all $\zeta \in L^{2}\left(0, T ; H^{1}(\Omega)\right)$ with $\partial_{t} \zeta \in L^{2}\left(\Omega_{T}\right)$ and $\zeta(T)=0$ and

$$
\int_{\Gamma_{T}}\left(\bar{\phi}_{N}-\phi_{0}\right) \partial_{t} \xi \mathrm{d} S(x) \mathrm{d} t=\int_{\Gamma_{T}} \nabla_{\Gamma} \mu_{\Gamma, N} \cdot \nabla \xi \mathrm{d} S(x) \mathrm{d} t
$$

for all $\xi \in L^{2}\left(0, T ; H^{1}(\Gamma)\right)$ with $\partial_{t} \xi \in L^{2}\left(\Gamma_{T}\right)$ and $\xi(T)=0$. Using the convergence properties from Lemma 4.7, we obtain the weak formulations (4.2) and (4.3) from Definition 4.1. Now, let $\eta \in L^{2}\left(0, T ; \mathcal{V}^{\kappa}\right) \cap$ $L^{\infty}\left(\Omega_{T}\right)$ be arbitrary. Testing (4.10c) with $\eta$ and integrating with respect to $t$ from 0 to $T$ we obtain that

$$
\begin{aligned}
\int_{\Omega_{T}} \nabla \phi_{N} \cdot \nabla \eta+F^{\prime}\left(\phi_{N}\right) \eta \mathrm{d}(x, t) & +\int_{\Gamma_{T}} \kappa \nabla_{\Gamma} \phi_{N} \cdot \nabla_{\Gamma} \eta+G^{\prime}\left(\phi_{N}\right) \eta \mathrm{d} S \mathrm{~d} t \\
& =\int_{\Omega_{T}} \mu_{N} \eta \mathrm{d}(x, t)+\int_{\Gamma_{T}} \mu_{\Gamma, N} \eta \mathrm{d} S \mathrm{~d} t .
\end{aligned}
$$

One can easily see that the terms that depend linearly on $\phi_{N}, \mu_{N}$ or $\mu_{\Gamma, N}$ are converging to the corresponding terms in (4.4) due to the convergence results that were established in Lemma 4.7. Recall that $G^{\prime}\left(\phi_{N}\right) \in$ $L^{1}\left(\Gamma_{T}\right)$ for all $N \in \mathbb{N}$ and $G^{\prime}\left(\phi_{N}\right) \rightarrow G^{\prime}(\phi)$ as $N \rightarrow \infty$ almost everywhere in $\Gamma_{T}$. Now, let $\varepsilon>0$ be arbitrary. For any $\alpha>0$, let $\Gamma_{\alpha}$ denote a measurable subset of $\Gamma_{T}$ with $\left|\Gamma_{\alpha}\right|<\alpha$. Using (P2) and the estimate $\int_{\Gamma} G\left(\phi_{N}(t)\right) \mathrm{d} S \leq E\left(\phi_{0}\right)+C^{*}$ we obtain that

$$
\begin{aligned}
& \int_{\Gamma_{\alpha}}\left|G^{\prime}\left(\phi_{N}\right)\right| \mathrm{d} S \mathrm{~d} t \\
& \quad \leq \delta T\left(E\left(\phi_{0}\right)+C^{*}\right)+\left(A_{G}^{\delta}+B_{G}\right)\left|\Gamma_{\alpha}\right|+B_{G} T^{1 / 2}\left|\Gamma_{\alpha}\right|^{1 / 2}\left\|\phi_{N}\right\|_{L^{\infty}\left(0, T ; L^{2}(\Gamma)\right)}
\end{aligned}
$$

for any $\delta>0$ where $C^{*}$ is the constant from (4.19). Fixing $\delta:=\varepsilon /\left[2 T\left(E\left(\phi_{0}\right)+C^{*}\right)\right]$ and assuming that $\alpha$ is sufficiently small, we obtain that

$$
\int_{\Gamma_{\alpha}}\left|G^{\prime}\left(\phi_{N}\right)\right| \mathrm{d} S \mathrm{~d} t \leq \frac{\varepsilon}{2}+\left(A_{G}^{\delta}+B_{G}\right) \alpha+B_{G} T^{1 / 2} \alpha^{1 / 2} C_{1}<\varepsilon .
$$

Therefore we can apply Vitali's convergence theorem (cf. [3, p. 57]) which implies that $G^{\prime}(\phi) \in L^{1}\left(\Gamma_{T}\right)$ with $G^{\prime}\left(\phi_{N}\right) \rightarrow G^{\prime}(\phi)$ in $L^{1}\left(\Gamma_{T}\right)$ and thus

$$
\int_{\Gamma_{T}} G^{\prime}\left(\phi_{N}\right) \eta \mathrm{d} S \mathrm{~d} t \rightarrow \int_{\Gamma_{T}} G^{\prime}(\phi) \eta \mathrm{d} S \mathrm{~d} t, \quad N \rightarrow \infty
$$

since $\eta \in L^{\infty}(\Gamma)$. The proof for $F^{\prime}(\phi) \in L^{1}\left(\Omega_{T}\right)$ and $\int_{\Omega} F^{\prime}\left(\phi_{N}\right) \eta \mathrm{d} x \rightarrow \int_{\Omega} F^{\prime}(\phi) \eta \mathrm{d} x$ proceeds analogously and we can finally conclude that the triple $\left(\phi, \mu, \mu_{\Gamma}\right)$ satisfies the weak formulations (4.2)-(4.4) of Definition 4.1. This means that we have verified item (ii) of the definition. 
Let now $t \in[0, T]$ be arbitrary. Then we have $\phi_{N}(t) \rightarrow \phi(t)$ almost everywhere on $\Omega$ and $\left.\left.\phi_{N}\right|_{\Gamma}(t) \rightarrow \phi\right|_{\Gamma}(t)$ almost everywhere on $\Gamma$. Moreover, recall that

$$
\left|F_{2}\left(\phi_{N}\right)\right| \leq C_{F}\left(1+\left|\phi_{N}\right|^{2}\right) \quad \text { in } \Omega \quad \text { and } \quad\left|G_{2}\left(\phi_{N}\right)\right| \leq C_{G}\left(1+\left|\phi_{N}\right|^{2}\right) \quad \text { on } \Gamma
$$

due to assumption (P2.4). Hence Lebesgue's general convergence theorem (cf. [3, p.60]) implies that

$$
\int_{\Omega} F_{2}\left(\phi_{N}(t)\right) \mathrm{d} x \rightarrow \int_{\Omega} F_{2}(\phi(t)) \mathrm{d} x \text { and } \int_{\Gamma} G_{2}\left(\phi_{N}(t)\right) \mathrm{d} S(x) \rightarrow \int_{\Gamma} G_{2}(\phi(t)) \mathrm{d} S(x)
$$

as $N \rightarrow \infty$. As the remaining terms of the energy $E$ are convex, we can conclude that

$$
\begin{aligned}
& E(\phi(t))+\frac{1}{2} \int_{0}^{t}\|\nabla \mu(s)\|_{L^{2}(\Omega)}^{2}+\left\|\nabla_{\Gamma} \mu_{\Gamma}(s)\right\|_{L^{2}(\Gamma)}^{2} \mathrm{~d} s \\
& \leq \liminf _{N \rightarrow \infty}\left\{E\left(\phi_{N}(t)\right)+\frac{1}{2} \int_{t-\tau}^{t}\left\|\nabla \mu_{N}(s)\right\|_{L^{2}(\Omega)}^{2}+\left\|\nabla_{\Gamma} \mu_{\Gamma, N}(s)\right\|_{L^{2}(\Gamma)}^{2} \mathrm{~d} s\right\} \\
& \leq E\left(\phi_{0}\right) .
\end{aligned}
$$

This verifies item (iii) of Definition 4.1 and completes the proof of Theorem 4.3.

\section{Uniqueness of the weak solution}

If the functions $F_{2}$ and $G_{2}$ that were introduced in (P2) are additionally Lipschitz continuous, we can even show that the weak solution predicted by Theorem 4.3 is unique:

Theorem 5.1. We assume that $\Omega \subset \mathbb{R}^{d}$ (with $d \in\{2,3\}$ ) is a bounded domain with Lipschitz boundary and that the potentials $F$ and $G$ satisfy the condition (P2). Let $T>0$ and $m=\left(m_{1}, m_{2}\right) \in \mathbb{R}^{2}$ be arbitrary and let $\phi_{0} \in \mathcal{V}_{m}^{\kappa}$ be any initial datum. Moreover, we assume that $F_{2}$ and $G_{2}$ are Lipschitz continuous. Then the weak solution $\left(\phi, \mu, \mu_{\Gamma}\right)$ that is predicted by Theorem 4.3 is the unique weak solution of the system (4.1).

Proof Let $\left(\phi, \mu, \mu_{\Gamma}\right)$ denote the solution that is given by Theorem 4.3. We assume that there is another weak solution $\left(\tilde{\phi}, \tilde{\mu}, \tilde{\mu}_{\Gamma}\right)$ of (4.1) in the sense of Definition 4.1 and we consider the difference

$$
\left(\bar{\phi}, \bar{\mu}, \bar{\mu}_{\Gamma}\right):=\left(\phi-\tilde{\phi}, \mu-\tilde{\mu}, \mu_{\Gamma}-\tilde{\mu}_{\Gamma}\right) .
$$

Due to (4.2) it holds that

$$
\int_{\Omega_{T}} \bar{\phi} \partial_{t} \zeta \mathrm{d}(x, t)=\int_{\Omega_{T}} \nabla \bar{\mu} \cdot \nabla \zeta \mathrm{d}(x, t)
$$

for all $\zeta \in L^{2}\left(0, T ; H^{1}(\Omega)\right)$ with $\partial_{t} \zeta \in L^{2}\left(\Omega_{T}\right)$ and $\zeta(T)=0$. For any $t_{0} \in[0, T]$ and any $\eta \in L^{2}\left(0, T ; \mathcal{V}^{\kappa}\right) \cap$ $L^{\infty}\left(\Omega_{T}\right)$ with $\left.\eta\right|_{\Gamma} \in L^{\infty}\left(\Gamma_{T}\right)$, we define

$$
\zeta(\cdot, t):= \begin{cases}\int_{t}^{t_{0}} \eta(\cdot, s) \mathrm{d} s & \text { if } t \leq t_{0} \\ 0 & \text { if } t>t_{0}\end{cases}
$$

Then, since $\zeta$ is a suitable test function, we have

$$
-\int_{\Omega_{t_{0}}} \bar{\phi} \eta \mathrm{d}(x, t)=\int_{\Omega_{t_{0}}} \nabla \bar{\mu} \cdot \nabla\left(\int_{t}^{t_{0}} \eta \mathrm{d} s\right) \mathrm{d}(x, t)=\int_{\Omega_{t_{0}}} \nabla\left(\int_{0}^{t} \bar{\mu} \mathrm{d} s\right) \cdot \nabla \eta \mathrm{d}(x, t) .
$$


This means that

$$
(-\Delta)^{-1} \bar{\phi}=-\int_{0}^{t} \bar{\mu} \mathrm{d} s+c \quad \text { and } \quad \partial_{t}(-\Delta)^{-1} \bar{\phi}=-\bar{\mu} .
$$

for some constant $c \in \mathbb{R}$. Now, choosing $\eta=\bar{\mu}$ yields

$$
\begin{aligned}
\int_{\Omega_{t_{0}}} \bar{\phi} \bar{\mu} \mathrm{d}(x, t) & =-\int_{\Omega_{t_{0}}} \nabla(-\Delta)^{-1} \bar{\phi} \cdot \nabla \partial_{t}(-\Delta)^{-1} \bar{\phi} \mathrm{d}(x, t) \\
& =-\frac{1}{2} \int_{\Omega_{t_{0}}} \frac{\mathrm{d}}{\mathrm{d} t}\left(\nabla(-\Delta)^{-1} \bar{\phi} \cdot \nabla(-\Delta)^{-1} \bar{\phi}\right) \mathrm{d}(x, t) \\
& =-\frac{1}{2} \int_{\Omega} \nabla(-\Delta)^{-1} \bar{\phi}\left(t_{0}\right) \cdot \nabla(-\Delta)^{-1} \bar{\phi}\left(t_{0}\right) \mathrm{d} x
\end{aligned}
$$

since $\bar{\phi}(0)=0$ and thus $(-\Delta)^{-1} \bar{\phi}(0)=0$. In a similar fashion, we can conclude that

$$
\int_{\Gamma_{t_{0}}} \bar{\phi} \bar{\mu}_{\Gamma} \mathrm{d}(x, t)=-\frac{1}{2} \int_{\Gamma} \nabla_{\Gamma}\left(-\Delta_{\Gamma}\right)^{-1} \bar{\phi}\left(t_{0}\right) \cdot \nabla_{\Gamma}\left(-\Delta_{\Gamma}\right)^{-1} \bar{\phi}\left(t_{0}\right) \mathrm{d} x .
$$

Summing up both equations gives

$$
\frac{1}{2}\left\|\bar{\phi}\left(t_{0}\right)\right\|_{\mathcal{V}_{0}^{\kappa *}}^{2}=-\int_{\Omega_{t_{0}}} \bar{\phi} \bar{\mu} \mathrm{d}(x, t)-\int_{\Gamma_{t_{0}}} \bar{\phi} \bar{\mu}_{\Gamma} \mathrm{d} S \mathrm{~d} t
$$

From the weak formulation (4.4) we obtain that

$$
\begin{aligned}
\int_{\Omega_{T}} \bar{\mu} \eta \mathrm{d}(x, t) & +\int_{\Gamma_{T}} \bar{\mu}_{\Gamma} \eta \mathrm{d} S \mathrm{~d} t=\int_{\Omega_{T}} \nabla \bar{\phi} \cdot \nabla \eta \mathrm{d}(x, t)+\int_{\Gamma_{T}} \kappa \nabla_{\Gamma} \bar{\phi} \cdot \nabla_{\Gamma} \eta \mathrm{d} S \mathrm{~d} t \\
& +\int_{\Omega_{T}}\left(F^{\prime}(\phi)-F^{\prime}(\tilde{\phi})\right) \eta \mathrm{d}(x, t)+\int_{\Gamma_{T}}\left(G^{\prime}(\phi)-G^{\prime}(\tilde{\phi})\right) \eta \mathrm{d} S \mathrm{~d} t
\end{aligned}
$$

for all $\eta \in L^{2}\left(0, T ; \mathcal{V}^{\kappa}\right) \cap L^{\infty}\left(\Omega_{T}\right)$ with $\left.\eta\right|_{\Gamma} \in L^{\infty}\left(\Gamma_{T}\right)$. Now, for any $M>0$, we choose the test function

$$
\eta:=\chi_{\left[0, t_{0}\right]} \mathcal{P}_{M}(\bar{\phi})
$$

where $\mathcal{P}_{M}$ describes a projection of $\mathbb{R}$ onto the interval $[-M, M]$ given by

$$
\mathcal{P}_{M}: \mathbb{R} \rightarrow \mathbb{R}, \quad s \mapsto\left\{\begin{array}{ll}
s & \text { if }|s|<M \\
\frac{s}{|s|} M & \text { if }|s| \geq M
\end{array}, \quad M>0\right.
$$

Recall that, according to $(\mathrm{P} 2), F=F_{1}+F_{2}$ and $G=G_{1}+G_{2}$ where $F_{1}$ and $G_{1}$ are convex. Hence, their derivatives $F^{\prime}$ and $G^{\prime}$ are monotonically increasing. In particular,

$$
\begin{aligned}
\left(F_{1}^{\prime}(\phi)-F_{1}^{\prime}(\tilde{\phi})\right) \mathcal{P}_{M}(\bar{\phi}) \geq 0 & \text { a.e. on } \Omega_{T} \\
\left(G_{1}^{\prime}(\phi)-G_{1}^{\prime}(\tilde{\phi})\right) \mathcal{P}_{M}(\bar{\phi}) \geq 0 & \text { a.e. on } \Gamma_{T} .
\end{aligned}
$$

Plugging these estimates into (5.2) with $\eta=\chi_{\left[0, t_{0}\right]} \mathcal{P}_{M}(\bar{\phi})$ gives

$$
\int_{\Omega_{t_{0}}} \bar{\mu} \mathcal{P}_{M}(\bar{\phi}) \mathrm{d}(x, t)+\int_{\Gamma_{t_{0}}} \bar{\mu}_{\Gamma} \mathcal{P}_{M}(\bar{\phi}) \mathrm{d} S \mathrm{~d} t
$$




$$
\begin{aligned}
& \geq \int_{\Omega_{t_{0}}} \nabla \bar{\phi} \cdot \nabla \mathcal{P}_{M}(\bar{\phi}) \mathrm{d}(x, t)+\int_{\Gamma_{t_{0}}} \kappa \nabla_{\Gamma} \bar{\phi} \cdot \nabla_{\Gamma} \mathcal{P}_{M}(\bar{\phi}) \mathrm{d} S \mathrm{~d} t \\
& \quad+\int_{\Omega_{t_{0}}}\left(F_{2}^{\prime}(\phi)-F_{2}^{\prime}(\tilde{\phi})\right) \mathcal{P}_{M}(\bar{\phi}) \mathrm{d}(x, t)+\int_{\Gamma_{t_{0}}}\left(G_{2}^{\prime}(\phi)-G_{2}^{\prime}(\tilde{\phi})\right) \mathcal{P}_{M}(\bar{\phi}) \mathrm{d} S \mathrm{~d} t .
\end{aligned}
$$

In the limit $M \rightarrow \infty$ we obtain (5.3) with $\chi_{\left[0, t_{0}\right]} \mathcal{P}_{M}(\bar{\phi})$ replaced by $\bar{\phi}$. Together with equation (5.1) this yields

$$
\begin{aligned}
& \frac{1}{2}\left\|\bar{\phi}\left(t_{0}\right)\right\|_{\mathcal{V}_{0}^{\kappa *}}^{2}+\|\nabla \bar{\phi}\|_{L^{2}\left(\Omega_{t_{0}}\right)}^{2}+\kappa\left\|\nabla_{\Gamma} \bar{\phi}\right\|_{L^{2}\left(\Gamma_{t_{0}}\right)}^{2} \\
& \quad \leq-\int_{\Omega_{t_{0}}}\left(F^{\prime}(\phi)-F^{\prime}(\tilde{\phi})\right) \bar{\phi} \mathrm{d}(x, t)-\int_{\Gamma_{t_{0}}}\left(G^{\prime}(\phi)-G^{\prime}(\tilde{\phi})\right) \bar{\phi} \mathrm{d} S \mathrm{~d} t \\
& \quad \leq L_{F}\|\bar{\phi}\|_{L^{2}\left(\Omega_{t_{0}}\right)}^{2}+L_{G}\|\bar{\phi}\|_{L^{2}\left(\Gamma_{t_{0}}\right)}^{2}
\end{aligned}
$$

where $L_{F}, L_{G} \geq 0$ are the Lipschitz costants of $F_{2}^{\prime}$ and $G_{2}^{\prime}$. Using integration by parts and Young's inequality with $0<\delta \leq 1$ we obtain that

$$
\|\bar{\phi}\|_{L^{2}(\Omega)}^{2}=\int_{\Omega} \nabla(-\Delta)^{-1} \bar{\phi} \cdot \nabla \bar{\phi} \mathrm{d} x \leq \frac{1}{4 \delta}\|\bar{\phi}\|_{\mathcal{V}_{0}^{\kappa *}}^{2}+\delta\|\nabla \bar{\phi}\|_{L^{2}(\Omega)}^{2} .
$$

We can bound $\|\bar{\phi}\|_{L^{2}(\Gamma)}^{2}$ using an interpolation inequality (see [20, Thm. II.4.1]). In combination with Poincaré's inequality and Young's inequality we infer that

$$
\|\bar{\phi}\|_{L^{2}(\Gamma)}^{2} \leq c\|\bar{\phi}\|_{L^{2}(\Omega)}^{2}+c\|\bar{\phi}\|_{L^{2}(\Omega)}\|\bar{\phi}\|_{H^{1}(\Omega)} \leq \frac{c}{\delta}\|\bar{\phi}\|_{L^{2}(\Omega)}^{2}+c \delta\|\nabla \bar{\phi}\|_{L^{2}(\Omega)}^{2}
$$

for any $0<\delta \leq 1$ and some generic constant $c \geq 0$ independent of $\delta$. Now, we apply (5.5) with $\delta^{2}$ instead of $\delta$ to obtain that

$$
\|\bar{\phi}\|_{L^{2}(\Gamma)}^{2} \leq \frac{\bar{c}}{\delta^{3}}\|\bar{\phi}\|_{\mathcal{V}_{0}^{\kappa *}}^{2}+\bar{c} \delta\|\nabla \bar{\phi}\|_{L^{2}(\Omega)}^{2}
$$

for any $0<\delta \leq 1$ and a constant $\bar{c} \geq 0$ independent of $\delta$. Then, inequality (5.4) implies that

$$
\begin{aligned}
& \frac{1}{2}\|\bar{\phi}\|_{\mathcal{V}_{0}^{\kappa *}}^{2}+\left(1-\left(L_{F}+\bar{c} L_{G}\right) \delta\right)\|\nabla \bar{\phi}\|_{L^{2}\left(\Omega_{t_{0}}\right)}^{2}+\kappa\left\|\nabla_{\Gamma} \bar{\phi}\right\|_{L^{2}\left(\Gamma_{t_{0}}\right)}^{2} \\
& \leq \frac{\frac{1}{4} L_{F}+\bar{c} L_{G}}{\delta^{3}} \int_{0}^{t_{0}}\|\bar{\phi}\|_{\mathcal{V}_{0}^{\kappa *}}^{2} \mathrm{~d} t .
\end{aligned}
$$

Now, choosing $\delta:=1 /\left(2 L_{F}+2 \bar{c} L_{G}\right)$ yields

$$
\left\|\bar{\phi}\left(t_{0}\right)\right\|_{\mathcal{V}_{0}^{\kappa *}}^{2} \leq C \int_{0}^{t_{0}}\|\bar{\phi}(t)\|_{\mathcal{V}_{0}^{\kappa *}}^{2}
$$

for some constant $C>0$ that depends only on $L_{F}, L_{G}$ and $\bar{c}$. Now, as $t_{0} \in[0, T]$ was arbitrary, Gronwall's lemma implies that

$$
\|\bar{\phi}(t)\|_{\mathcal{V}_{0}^{\kappa *}}^{2}=0
$$

for almost all $t \in[0, T]$. Thus, $\phi=\tilde{\phi}$ almost everywhere on $\Omega_{T}$ and $\left.\phi\right|_{\Gamma}=\left.\tilde{\phi}\right|_{\Gamma}$ almost everywhere on $\Gamma_{T}$. Recall that both $\left(\phi, \mu, \mu_{\Gamma}\right)$ and $\left(\tilde{\phi}, \tilde{\mu}, \tilde{\mu}_{\Gamma}\right)$ satisfy the weak formulation (4.4). Choosing $\eta \in C_{c}^{\infty}(\Omega)$ we obtain that

$$
\int_{\Omega_{T}}(\mu-\tilde{\mu}) \eta \mathrm{d} x=\int_{\Omega_{T}} \nabla \phi \cdot \nabla \eta+F^{\prime}(\phi) \eta \mathrm{d} x-\int_{\Omega_{T}} \nabla \tilde{\phi} \cdot \nabla \eta+F^{\prime}(\tilde{\phi}) \eta \mathrm{d} x=0
$$


since $\phi=\tilde{\phi}$ almost everywhere in $\Omega_{T}$. Thus, $\mu=\tilde{\mu}$ almost everywhere in $\Omega$ directly follows. Testing (4.4) with $\eta \in C^{\infty}(\Omega)$ and using (5.6) gives

$$
\begin{aligned}
& \int_{\Gamma_{T}} \mu_{\Gamma} \eta \mathrm{d} S-\int_{\Gamma_{T}} \tilde{\mu}_{\Gamma} \eta \mathrm{d} S \\
& \quad=\int_{\Gamma_{T}} \kappa \nabla_{\Gamma} \phi \cdot \nabla_{\Gamma} \eta+G^{\prime}(\phi) \eta \mathrm{d} x-\int_{\Gamma_{T}} \kappa \nabla_{\Gamma} \tilde{\phi} \cdot \nabla_{\Gamma} \eta+G^{\prime}(\tilde{\phi}) \eta \mathrm{d} x=0 .
\end{aligned}
$$

This implies that $\mu_{\Gamma}=\tilde{\mu}_{\Gamma}$ almost everywhere on $\Gamma$ and completes the proof of Theorem 5.1.

Remark 5.2. Since the unique weak solution from Theorem 5.1 exists on the interval $[0, T]$ for any given $T>0$, it can be considered as the unique weak solution on $[0, \infty[$. This means that this solution is global in time.

\section{$6 \quad$ Numerical results}

In this section we present several plots of two numerical simulations (see Figure 1 and Figure 2) for the system (1.13) in two dimensions with $\varepsilon=0.02$. These numerical solutions were computed by a finite element method implemented by D. Trautwein [34] using MATLAB. In both simulations, the domain $\Omega$ is a square that is discretized by a standard Friedrichs-Keller triangulation with step size $h$. Therefore we write $\Omega_{h}$ and $\Gamma_{h}$ to denote the discretizations of $\Omega$ and $\Gamma$. The time evolution is computed by an implicit Euler method with a step size of $\varepsilon^{3}=8 \cdot 10^{-6}$.

In the first simulation, the parameter $\kappa$ is set to 0.02 and the domain $\Omega$ is the unit square. The spatial step size for the Friedrichs-Keller triangulation is $h=0.01$ which leads to a grid of $101 \times 101$ sampling points. Figure 1 visualizes the time evolution of an initial datum $\phi_{0}$ that is set to zero in every node of $\Omega_{h}$ but set to one in every node of $\Gamma_{h}$. The plots show the solution after 5, 15, 80, 200, 500 and 2000 time steps. Note that, as the mass on the boundary is conserved, the solution will remain identically one on the boundary for all time. The phase separation in the bulk leads to a wave-like structure and eventually, as a long-time effect, the pure phase associated with the value -1 forms a single circle around the center of the square $\Omega$.

In the second simulation, we use the parameter $\kappa=0.075$ and the domain $\Omega=] 0,0.5\left[^{2}\right.$. The spatial step size is $h=0.005$ and therefore the grid also consists of $101 \times 101$ sampling points. Now, the initial datum $\phi_{0}$ attains random values between -0.1 and 0.1 in every grid point of $\Omega_{h}$ and also random values between 0.4 and 0.6 in every node of $\Gamma_{h}$. Figure 2 shows the corresponding solution after 5, 15, 50, 125, 300 and 2500 time steps. 

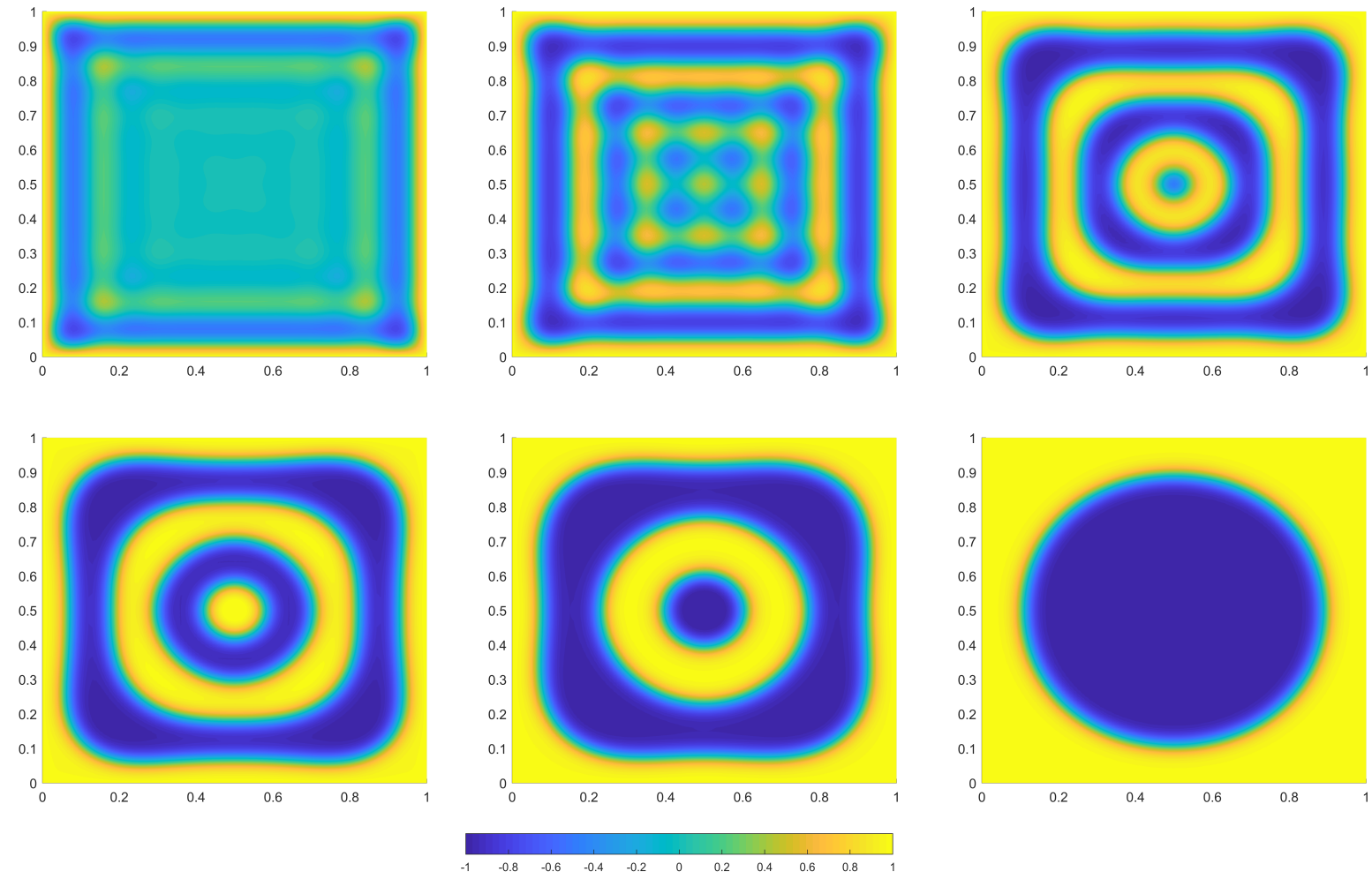

Figure 1: The initial datum $\phi_{0}$ is set to zero in $\Omega_{h}$ and set to one on $\Gamma_{h}$.
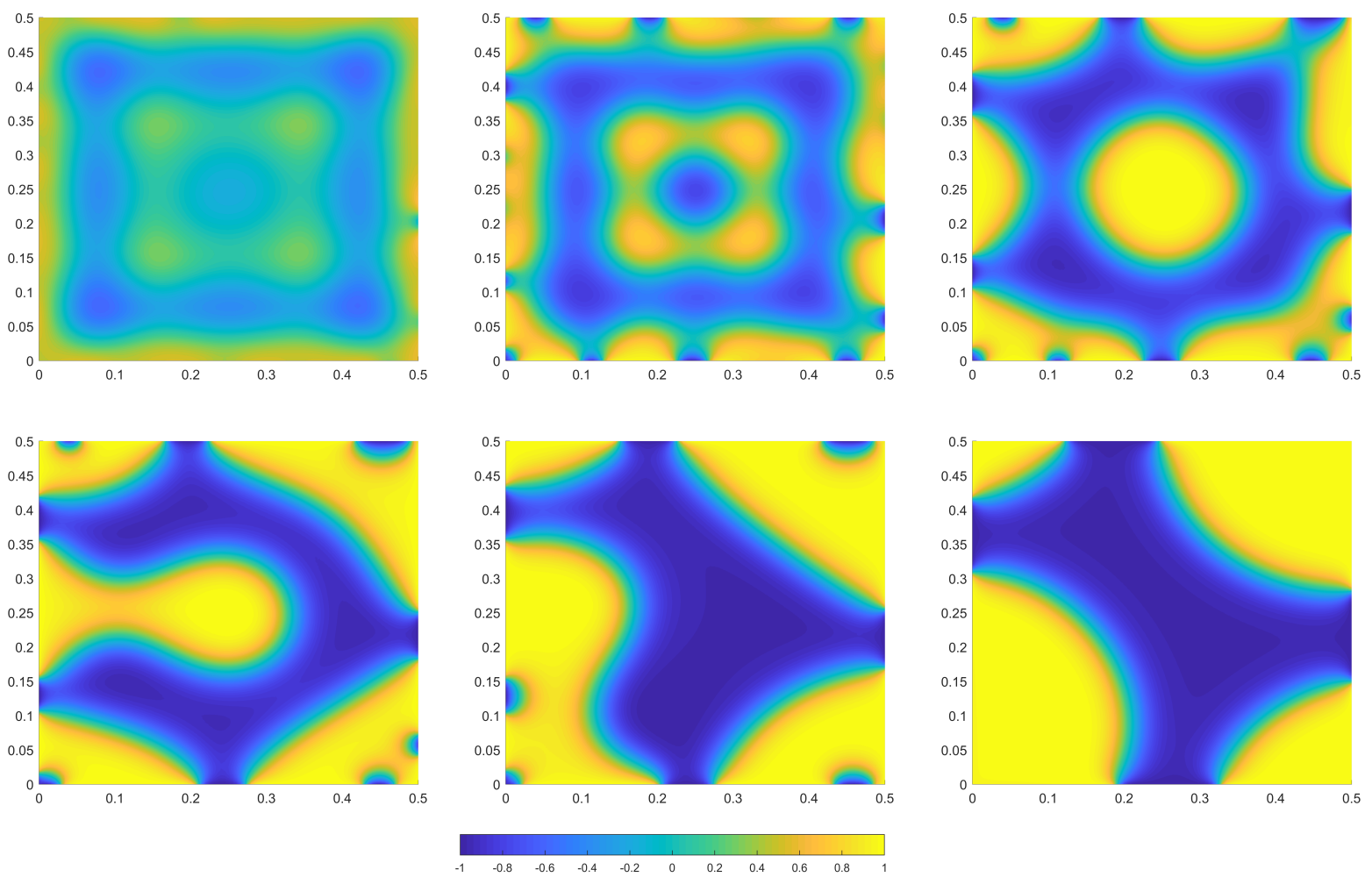

Figure 2: The initial datum $\phi_{0}$ attains random values between -0.1 and 0.1 in $\Omega_{h}$ and random values between 0.4 and 0.6 on $\Gamma_{h}$. 


\section{Appendix}

For the reader's convenience, we state the generalized Poincaré inequality as presented by H.W. Alt [3, p. 242] because of its importance in the approach of Section 4.4:

Lemma 6.1 (Generalized Poincaré inequality). Let $\Omega \subset \mathbb{R}^{n}$ be open, bounded and connected with Lipschitz boundary $\partial \Omega$. Moreover, let $1<p<\infty$ and let $\mathcal{M} \subset W^{1, p}(\Omega)$ be nonempty, closed and convex. Then the following items are equivalent for every $u_{0}$ in $\mathcal{M}$ :

(i) There exists a constant $C_{0}<\infty$ such that for all $\xi \in \mathbb{R}$,

$$
u_{0}+\xi \in \mathcal{M} \Rightarrow|\xi| \leq C_{0}
$$

(ii) There exists a constant $C<\infty$ with

$$
\|u\|_{L^{p}(\Omega)} \leq C\left(\|\nabla u\|_{L^{p}(\Omega)}+1\right) \quad \text { for all } u \in \mathcal{M}
$$

\section{References}

[1] H. Abels and M. Wilke. Convergence to equilibrium for the Cahn-Hilliard equation with a logarithmic free energy. Nonlinear Anal., 67:3176-3193, 2007.

[2] S.M. Allen and J.W. Cahn. A microscopic theory for the antiphase boundary motion and its application to antiphase domain coarsening. Acta Met., 27(6):1085-1095, 1979.

[3] H.W. Alt. Linear Functional Analysis - An Application-Oriented Introduction. Springer, London, 2016.

[4] L. Ambrosio, N. Gigli, and G. Savare. Gradient Flows in Metric Spaces and in the Space of Probability Measures. Birkhäuser Basel, 2008.

[5] P. Bates and P. Fife. The dynamics of nucleation for the Cahn-Hilliard equation. SIAM J. Appl. Math., 53(4):990-1008, 1993.

[6] C. Liu and H. Wu. An Energetic Variational Approach for the Cahn-Hilliard Equation with Dynamic Boundary Condition: Model Derivation and Mathematical Analysis. Arch. Rational Mech. Anal., https://doi. org/10. 1007/ s00205-019-01356-x, 2019.

[7] J.W. Cahn and J.E. Hilliard. Free energy of a nonuniform system I. Interfacial free energy. J. Chem. Phys., 2:205-245, 1958.

[8] L. Cherfils, S. Gatti, and A. Miranville. A variational approach to a Cahn-Hilliard model in a domain with nonpermeable walls. Problems in mathematical analysis. J. Math. Sci., 189(4):604-636, 2013.

[9] L. Cherfils, A. Miranville, and S. Zelik. The Cahn-Hilliard equation with logarithmic potentials. Milan J. Math., 79:561$596,2011$.

[10] R. Chill, E. Făsangová, and J. Prüss. Convergence to steady state of solutions of the Cahn-Hilliard and Caginalp equations with dynamic boundary conditions. Math. Nachr., 279(13-14):1448-1462, 2006.

[11] P. Colli and T. Fukao. The Allen-Cahn equation with dynamic boundary conditions and mass constraints. Math. Meth. Appl. Sci., 38(17):3950-3967, 2014.

[12] P. Colli and T. Fukao. Cahn-Hilliard equation with dynamic boundary conditions and mass constraint on the boundary. J. Math. Anal. Appl., 429(2):1190-1213, 2015.

[13] P. Colli, T. Fukao, and K.F. Lam. On a coupled bulk-surface Allen-Cahn system with an affine linear transmission condition and its approximation by a Robin boundary condition. Nonlinear Anal., 184:116-147, 2019.

[14] P. Colli, G. Gilardi, R. Nakayashiki, and K. Shirakawa. A class of quasi-linear Allen-Cahn type equations with dynamic boundary conditions. Nonlinear Anal., 158:32-59, 2017.

[15] P. Colli, G. Gilardi, and J. Sprekels. On the Cahn-Hilliard equation with dynamic boundary conditions and a dominating boundary potential. J. Math. Anal. Appl., 419(2):972-994, 2014.

[16] C.M. Elliott and H. Garcke. On the Cahn-Hilliard equation with degenerate mobility. SIAM J. Math. Anal., 27:404-424, 1996.

[17] C.M. Elliott and S. Zheng. On the Cahn-Hilliard equation. Arch. Rational Mech. Anal., 96:339-357, 1986.

[18] C.G. Gal. A Cahn-Hilliard model in bounded domains with permeable walls. Math. Methods App. Sci., 29:2009-2036, 2006.

[19] C.G. Gal and H. Wu. Asymptotic behavior of a Cahn-Hilliard equation with Wentzell boundary conditions and mass conservation. Discrete Contin. Dyn. Syst., 22:1041-1063, 2008. 
[20] G.P. Galdi. An introduction to the mathematical theory of the Navier-Stokes equations. Springer Monographs in Mathematics. Springer, New York, second edition, 2011.

[21] H. Garcke. On Cahn-Hilliard systems with elasticity. Proc. Roy. Soc. Edinburgh, 133 A:307-331, 2003.

[22] G.R. Goldstein, A. Miranville, and G. Schimperna. A Cahn-Hilliard model in a domain with non permeable walls. Physica $D, 240: 754-766,2011$.

[23] M. Grinfeld and A. Novick-Cohen. Counting stationary solutions of the Cahn-Hilliard equation by transversality arguments. Proc. Roy. Soc. Edinburgh Sect. A, 125:351-370, 1995.

[24] R. Kenzler, F. Eurich, P. Maass, B. Rinn, J. Schropp, E. Bohl, and W. Dietrich. Phase separation in confined geometries: Solving the Cahn-Hilliard equation with generic boundary conditions. Comp. Phys. Comm., 133:139-157, 2001.

[25] M. Liero. Passing from bulk to bulk-surface evolution in the Allen-Cahn equation. Nonlinear Differ. Equ. Appl., 20:919942, 2013.

[26] R.M. Mininni, A. Miranville, and S. Romanelli. Higher-order Cahn-Hilliard equations with dynamic boundary conditions. J. Math. Anal. Appl., 449(2):1321-1339, 2017.

[27] A. Miranville and S. Zelik. The Cahn-Hilliard Equation with Singular Potentials and Dynamic Boundary Conditions. Discrete and Continuous Dynamical Systems, 28(1):275-310, 2010.

[28] T. Motoda. Time periodic solutions of Cahn-Hilliard systems with dynamic boundary conditions. AIMS Math., 3(2):263$287,2018$.

[29] R. Pego. Front migration in the nonlinear Cahn-Hilliard equation. Proc. R. Soc. Lond. A, 422:261-278, 1989.

[30] R. Racke and S. Zheng. The Cahn-Hilliard equation with dynamic boundary conditions. Adv. Differential Equations, 8(1):83-110, 2003.

[31] P. Rybka and K.H. Hoffmann. Convergence of solutions to Cahn-Hillard equation. Commun. Partial Differential Equations, 24(5-6):1055-1077, 1999.

[32] J. Simon. Compact sets in the space $L^{p}(0, T ; B)$. Annali di Matematica Pura ed Applicata, 146:65-96, 1986.

[33] P.A. Thompson and M.O. Robbins. Simulations of contact-line motion: slip and the dynamic contact angle. Phys. Rev. Lett., 63:766-769, 1989.

[34] D. Trautwein. Finite-Elemente Approximation der Cahn-Hilliard-Gleichung mit Neumann- und dynamischen Randbedingungen. Bachelor thesis, University of Regensburg, 2018.

[35] H. Wu and S. Zheng. Convergence to equilibrium for the Cahn-Hilliard equation with dynamic boundary conditions. J. Differential Equations, 204(2):511-531, 2004.

[36] S. Zheng. Asymptotic behavior of solution to the Cahn-Hillard equation. Appl. Anal., 23(3):165-184, 1986. 